\title{
L'instrumentalisation du passé en Grèce dans la mobilisation de la jeunesse par le régime du 4 août : octobre 1940-avril 1941
}

How the past is instrumentalized in Greece to the youth by the Metaxas regime:

October 1940-April 1941

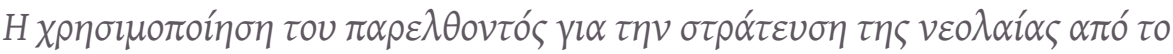

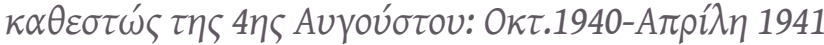

\section{Christophe Chalas}

\section{OpenEdition}

Journals

Édition électronique

URL : https://journals.openedition.org/ceb/3984

DOI : $10.4000 / c e b .3984$

ISSN : 2261-4184

\section{Éditeur}

INALCO

Édition imprimée

Pagination : 271-300

ISBN : 978-2-85831-205-4

ISSN : 0290-7402

\section{Référence électronique}

Christophe Chalas, « L'instrumentalisation du passé en Grèce dans la mobilisation de la jeunesse par le régime du 4 août : octobre 1940-avril 1941 », Cahiers balkaniques [En ligne], 41 | 2013, mis en ligne le 19 mai 2013, consulté le 06 juillet 2021. URL : http://journals.openedition.org/ceb/3984 ; DOI : https:// doi.org/10.4000/ceb.3984

Ce document a été généré automatiquement le 6 juillet 2021.

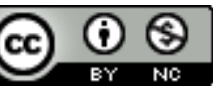

Cahiers balkaniques est mis à disposition selon les termes de la Licence Creative Commons Attribution - Pas d'Utilisation Commerciale 4.0 International. 


\section{L'instrumentalisation du passé en Grèce dans la mobilisation de la jeunesse par le régime du 4 août : octobre 1940-avril 1941}

How the past is instrumentalized in Greece to the youth by the Metaxas regime:

October 1940-April 1941

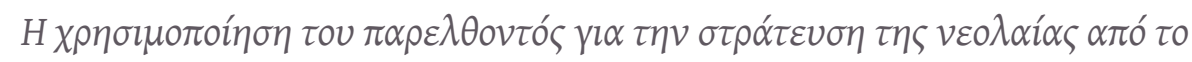

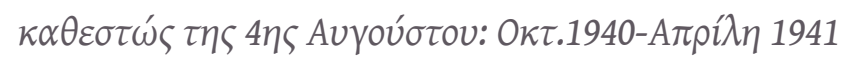

\section{Christophe Chalas}

\section{Introduction}

1 Cet article s'inscrit dans une triple dynamique que connaissent depuis un certain nombre d'années la recherche historique et les études grecques. La première a vu et voit encore se multiplier les travaux sur les usages idéologique et politique de l'histoire et du passé. La seconde concerne un intérêt renouvelé et, en partie, "dépassionné » pour la période de l'histoire grecque correspondant au régime du 4 août (1936-1941) qui a été souvent étudiée sous le prisme de considérations idéologiques ${ }^{1}$. La troisième enfin, initiée déjà depuis un certain temps a fait de la jeunesse et notamment de l'EON, un objet d'étude de plus en plus interrogé dans l'historiographie hellénique ${ }^{2}$. Ces orientations se sont ainsi traduites notamment par de nombreux travaux portant sur les programmes, les manuels scolaires, l'enseignement de l'histoire. Cet article s'inscrit donc dans cette triple orientation.

2 C'est dans un contexte d'instabilité institutionnelle et politique chronique et en raison d'une supposée menace d'un coup de force communiste qu'avec la bénédiction du roi Georges II, le Premier ministre Ioannis Métaxas ${ }^{3}$ dissout le Parlement, suspend les libertés fondamentales et interdit les partis politiques le 4 août 1936, date qui donnera 
son nom au régime. La dictature métaxo-monarchique qui se met en place entame alors une politique de répression des oppositions qui touche en particulier les communistes, le régime amplifiant ainsi un arsenal juridique qui préexistait assez largement tandis que l'exil et l'incarcération dans des camps îliens écartent du corps social les «mauvais citoyens ${ }^{4}$. Néanmoins, inscrit dans « l'âge totalitaire ", le régime du général Métaxas ne vise pas seulement à éradiquer les "menaces " qui pèseraient sur la nation et l'identité grecques, mais aussi à forger une société nouvelle et régénérée. La jeunesse constitue donc un matériau fondamental et il ne fait pas mystère de l'extrême intérêt qu'il lui porte 5 .

3 Dans la mesure où le régime met en place un certain nombre de réformes éducatives, en imposant la langue démotique dans l'enseignement primaire, en élaborant de nouveaux programmes et en instaurant pour la première fois dans l'histoire du pays un organisme étatique de publication de manuels scolaires ${ }^{6}$, il manifeste clairement l'ambition d'un contrôle plus abouti de l'enseignement. Néanmoins, à la fin des années $1930,90 \%$ des enfants sortent du système scolaire à la fin du cycle primaire ${ }^{7}$ et échappent donc à ce potentiel endoctrinement. Ainsi, incontestablement inspirée par les modèles fasciste et nazi, l'ordonnance du 7 novembre 1936 instaure l'Organisation nationale de la Jeunesse (EON) dont les buts sont « de consacrer profitablement le temps libre en dehors du travail et des études à la promotion de la condition physique et culturelle, au développement du sentiment national et de la foi religieuse, en créant une collaboration spirituelle et une solidarité sociale $~^{8}$. En 1939 , les organisations chrétiennes de scoutisme sont dissoutes, et l'EON des phalangistes et pionniers en uniforme bleu et blanc devient progressivement une organisation de masse ${ }^{9}$. Le régime affiche donc une volonté de façonner corps et esprit de la jeunesse hellénique.

4 La politique d'«armement idéologique» de la jeunesse (selon la phraséologie de l'époque) et la recherche de son adhésion au régime conduisent à la création de

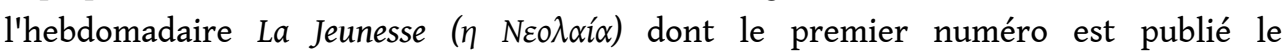
15 octobre 1938. Jusqu'en avril $1941^{10}$ paraîtront 133 numéros de "L'organe de formation spirituelle, religieuse, morale, sociale et politique des jeunes ${ }^{11}$ qui constituent le vecteur premier de la «catéchèse " gouvernementale. Cet article veut explorer ce matériau selon trois axes principaux : montrer comment le passé historique ou mythique éclaire la lecture des enjeux du conflit et alimente la définition du Nous et de l'Autre, repérer comment des modèles historiques alimentent le rôle assigné aux jeunes, explorer en quoi le contexte et «l'écriture de l'histoire » qui en découle peuvent constituer une "opportunité » pour le régime du 4 août dans la réalisation d'objectifs, dont la dimension totalitaire sera interrogée.

\section{La poursuite de l'« épopée grecque »: Nous et l'Autre}

Face à la montée des périls à partir de 1936, le régime du 4 août est confronté à une certaine tension au sommet de l'État. Le monarque Georges II incarne les positions anglophiles alors que Métaxas, par sa culture et sa formation militaire, regarde davantage vers Berlin. Néanmoins, "le pays réussissait à maintenir une fragile ligne médiane entre ces deux tendances a priori contradictoires par une politique fort prudente ${ }^{12}$ qui n'exclut pas la conviction progressivement construite chez le général que les intérêts grecs doivent maintenir le pays dans le giron anglais. Ainsi la Grèce se maintient dans le camp des forces « conservatrices » que sont la France ou le Royaume- 
Uni, face aux États "révisionnistes" dont les prétentions inquiètent Athènes, en particulier les visées italiennes et bulgares. Il lui faut donc ne jamais adopter une position qui pourrait être jugée provocatrice ${ }^{13}$ tout en préparant une guerre qui semble

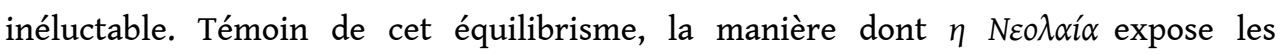
événements de septembre 1939: vis-à-vis de la Pologne envahie, "il ne s'agit pas de montrer ici sympathie ou antipathie ", mais le rédacteur affirme que la Grèce défend "la souveraineté et l'indépendance de tous les peuples " alors que « les petits États dont les peuples veulent seulement se consacrer à leur labeur pacifique n'ont pas et ne veulent pas d'ennemis". Mais il "existe un risque perceptible qu'une vague de barbarie s'abatte sur le monde» (N. 2.9.1939, p.1513, 9.9.1939, p.1546-1547). Les allusions sont transparentes : la Grèce n'est pas du côté de la brutalité impérialiste.

6 Toutefois, la prudence ne permet pas au pays d'échapper au conflit : le 28 octobre 1940, le «'OXl» de Métaxas à l'ultimatum italien précipite la Grèce dans la Seconde Guerre mondiale. L'offensive italienne depuis l'Albanie se porte vers l'Épire et, non seulement l'armée grecque réussit à stopper l'ennemi entre le 28 octobre et le 13 novembre 1940, mais elle lance une contre-offensive qui lui permet d'entrer en territoire albanais et d'occuper l'Épire du Nord. Dans des conditions hivernales terribles, alors que «la campagne d'Albanie est un calvaire pour les deux camps", "les Grecs en font une épopée » ${ }^{14}$

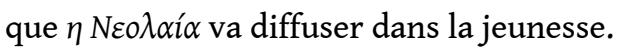

\section{Un modèle ancestral et édifiant de comportement}

7 La mobilisation de la jeunesse grecque et les sacrifices qui lui sont demandés trouvent leur légitimation dans un panthéon des ancêtres-héros, l'exemplarité d'actes, d'attitudes, mais aussi de formules puisées dans l'histoire ou le mythe. C'est selon l'expression de Michael Hertzfeld une "généalogie impeccable " et supposée continue qui doit donner son sens au combat ${ }^{15}$. D'Héraclès à Botsaris en passant par Achille, Léonidas, Thémistocle, Alexandre ${ }^{16}$, Basile le Bulgaroctone, Constantin Paléologue, Germanos de Patras et Kolokotronis, la mythologie, le poème épique et l'histoire sont convoqués pour célébrer la geste héroïque des aïeux de la jeunesse hellénique ( $\mathrm{N}$. 30.11 .1940$, p. 242) qui se voit sommée d'être digne de modèles qui incarnent l'idéal hellénique de défense de la patrie et de la liberté.

8 Mais, au-delà de ces figures tutélaires, le combat « éternel » des Grecs est aussi celui du

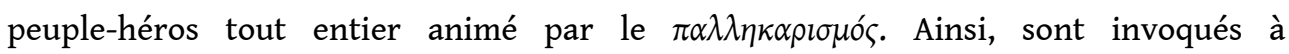
d'innombrables reprises l'héroïsme des Trois Cents qui «demeurent le symbole du patriotisme grec» (N. 9.11.1940 p.180) et celui des combattants antiques, car, alors que «Les Perses attendaient la victoire [...] ce furent Platées, Salamine, Marathon 》 (N. 2.11.1940, p.138). Le bataillon des hoplites, la phalange macédonienne côtoient les Akrites, les Pallikares et les soldats des guerres balkaniques (N. 30.11.1940, p. 242 et 2.11.1940, p. 141) dans un défilé ininterrompu de faits d'armes, et d'héroïsme grec. Mais l'évocation maintes fois répétée de la «danse de Zalongo », des " assiégés libres de Missolonghi » (N. 2.11.1940, p. 132) ou du drame du monastère d'Arkadi associe l'ensemble du peuple grec dans ses innombrables combats et les sacrifices exemplaires qu'il a consentis. C'est, selon les mots de Michaël Hertzfeld, "l'incorporation du laos dans l'ethnos historique ${ }^{17}$ qui se traduit aussi par ces mots : "Lundi dernier, les chefs du peuple grec ont atteint le sommet du sens de l'histoire hellénique [...] et le peuple à son tour est venu au sommet de son devoir pour continuer cette histoire» (N. 2.11.1940, p. 132). 
Bravoure et sacrifice exaltés des héros et du peuple grec s'incarnent dans une geste, indissociable du verbe. Les rédacteurs de la revue (ainsi que Métaxas, son successeur Koryzis ou le roi Georges II dont de nombreux messages à la nation sont publiés) parsèment leur propos des formules-symboles de la bravoure et de la résistance grecques. "Viens les prendre ${ }^{18}$ », entendrez-vous la voix de Léonidas et des Trois Cents quand ils décidèrent de mourir aux Thermopyles, "Nous ne te remettrons pas la ville» entendrez vous affirmer Constantin [...] «La Liberté ou la Mort » entendrez vous crier les héros de la Révolution [...] La même phrase avec des mots différents, mais toujours le même sens" (N.5.04.1941, p. 675). Ces mots sont autant de «Non » jalonnant le passé qui font écho au «'OXl» de Métaxas le 28 octobre 1940 inscrit ainsi dans cette continuité verbale. Verbe mobilisateur aussi la parole littéraire et poétique, d'Eschyle à Palamas, les mots du tragique ornent la couverture de la revue et accompagnent le portrait et les paroles du chef, tandis que la filiation avec les combats révolutionnaires de 1821 surgit sous la plume de l'écrivain contemporain et se voit renforcée par l'image ${ }^{19}$.

Ainsi, l'histoire grecque apparaît-elle comme une épopée ancestrale encourageant la mobilisation des jeunes qui ont "une dette envers le passé» (N.14.12.1940, p. 289) et doivent se montrer dignes de ce lourd héritage. Le Nous qui se dessine ici établit un continuum historique (N. 23.11.1940, p. 230) entre les Grecs du passé et ceux de 1940-41, entre les actes des glorieux ancêtres et ceux du présent, entre les morts et les vivants. Dessinant une histoire et une géographie sacrificielles, l'exaltation nationaliste qui s'ancre ainsi dans la terre et les morts prend des accents barrésiens ${ }^{20}$. Or, si la jeunesse grecque sait de quels modèles elle doit s'inspirer, elle doit aussi être convaincue de ce pour quoi elle doit se mobiliser.

Couvertures de la revue des 2 et 23 novembre 1940

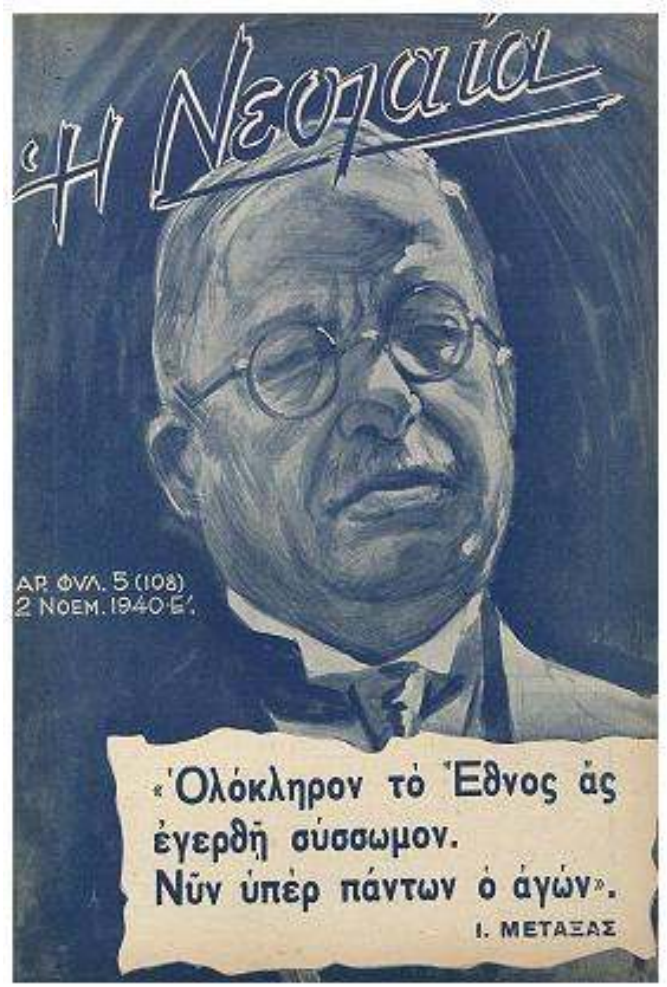

imagehttp://picasaweb.google.com/109214261579697097237/21940\#5478510822708315250 


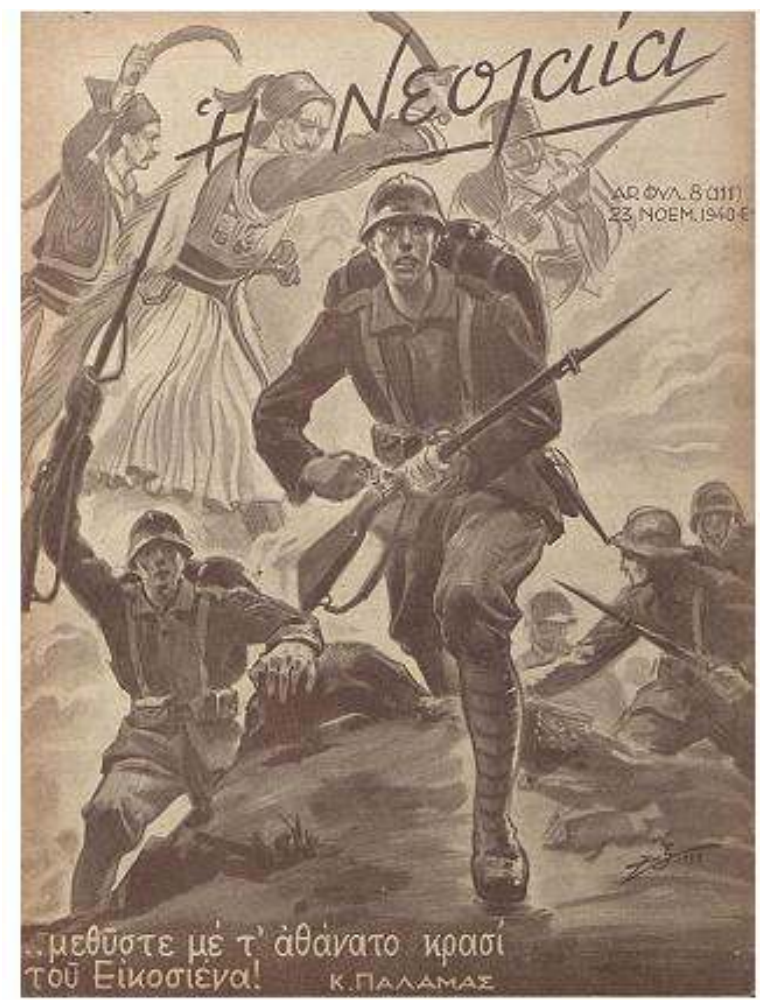

http://picasaweb.google.com/109214261579697097237/231940\#5478514860846583858

\section{Une guerre patriotique et un conflit de civilisation}

11 Le premier devoir qui s'impose à la jeunesse, forte de la conviction que « Nous avons une arme secrète avec laquelle nous combattons depuis toujours l'ennemi plus grand [...] C'est l'amour de la Grèce et de la Liberté » (N. 29.03.1941, p. 669), est la défense d'un territoire et de son indépendance menacés. Les arguments doivent ancrer chez le jeune lecteur la conviction que cette terre est grecque de toute éternité et que ce territoire est borné par la grécité séculaire de ce sol sacré (N. 9.11.1940, p. 161 et 30.11.1940, p. 254) . Ainsi s'observe dans le discours la mise en équation de la «race " grecque et du territoire (historiquement mouvant d'ailleurs) sur lequel elle s'inscrit par la promotion d'une forme de déterminisme "racialo-géopolitique $»^{21}$. Les jeunes doivent montrer qu'ils sont « dignes de la liberté que (leur) ont donnée (leurs) ancêtres ». 1821 et la révolution où la Grèce "a ressuscité de son tombeau » (N. 29.03.1941, p.653) constituent l'événement fondateur dont le souffle doit animer l'EON, mais qu'ils doivent même dépasser, car c'est «un combat plus important peut-être que 1821, car nous combattions alors pour détruire le joug alors que nous nous battons pour ne pas perdre la liberté arrachée au prix de tant de sang » (N. 21.12.1940, p. 320).

Passé et histoire exemplaires constituent donc les moteurs du patriotisme, mais le combat dépasse ces horizons : " la route séculaire de notre Race, son destin privilégié [...] (est de) sauver non seulement sa Patrie, mais aussi l'Humanité" (N.9.11.1940, p.163). La propagande du régime dépasse le caractère territorial et géopolitique du conflit pour en faire une guerre de civilisation. Elle s'emploie à convaincre du devoir de combattre un ennemi qui bafoue la justice et le droit en déclenchant une agression illégitime et impérialiste qui contraste avec l'histoire sans tache des Grecs puisque «toujours des 
guerres défensives (ont été) menées par les Grecs, jamais pour asservir les autres. ${ }^{22} \ggg$. Promesse est faite à la jeunesse que les sacrifices exigés d'elle verront le triomphe de ces valeurs (N.1.02.1941, p. 1).

13 Le clivage majeur qui peuple d'innombrables articles oppose civilisation et barbarie et fait de la Grèce, « encore une fois en première ligne de l'histoire » et dont « le destin [...] a été depuis l'aube de l'histoire de s'opposer aux barbares et de les vaincre» (N. 2.11.1940, p. 132), une sentinelle infatigable. C'est parce que ce pays est le «berceau de la civilisation » (N.7.12.1940, p. 280 et 15.02.1941, p. 520), « la patrie de Prométhée qui fit le don du feu aux bandes barbares des premiers hommes " (N. 26.04.1941, p. 748) que son passé est porteur d'une dimension messianique. La Grèce est la " gardienne du temple " civilisé et sans elle le chaos barbare ${ }^{23}$, se serait imposé. Il faut convaincre que la guerre qui oppose les Grecs aux Italiens est de même nature que les guerres médiques ou la guerre d'indépendance face à l'Empire ottoman. Comme à Marathon ou Salamine, «sans (lesquels) les Asiatiques auraient asservi l'Europe » (N.11.01.1941, p. 395), dans les montagnes du Pinde ou en Macédoine, se joue une tragédie aux accents eschatologiques contre une «barbarie dégénérée » (N. 11.01.1941, p. 395).

La propagande martèle aussi les différences entre le Nous et l'Autre sur le terrain plus prosaïque d'une forme de civilité. Puisque «le caractère chevaleresque des combattants de Marathon et de Sparte vit à nouveau» (N.1.02.1941, p. 467) dans la façon humaine et civilisée dont sont traités les prisonniers italiens, les jeunes Phalangistes et Pionniers doivent se montrer les dignes héritiers des irréprochables ancêtres ${ }^{24}$, si la force mécanique et numérique est du côté de l'ennemi, l'âme et sa grandeur sont grecques ! De la faiblesse objective des modestes forces militaires, humaines ${ }^{25}$ et matérielles de la Grèce, la propagande du régime fait inlassablement un levier de mobilisation : l'âme grecque permet de lutter "les mains nues contre la mécanique d'acier", tel un nouveau "David contre Goliath » (N. 9.11.1940, p. 163). Les convictions idéalistes des hommes du 4 août, qui jusqu'à lors alimentaient le combat anticommuniste, vont nourrir la mobilisation contre les forces de l'Axe. 


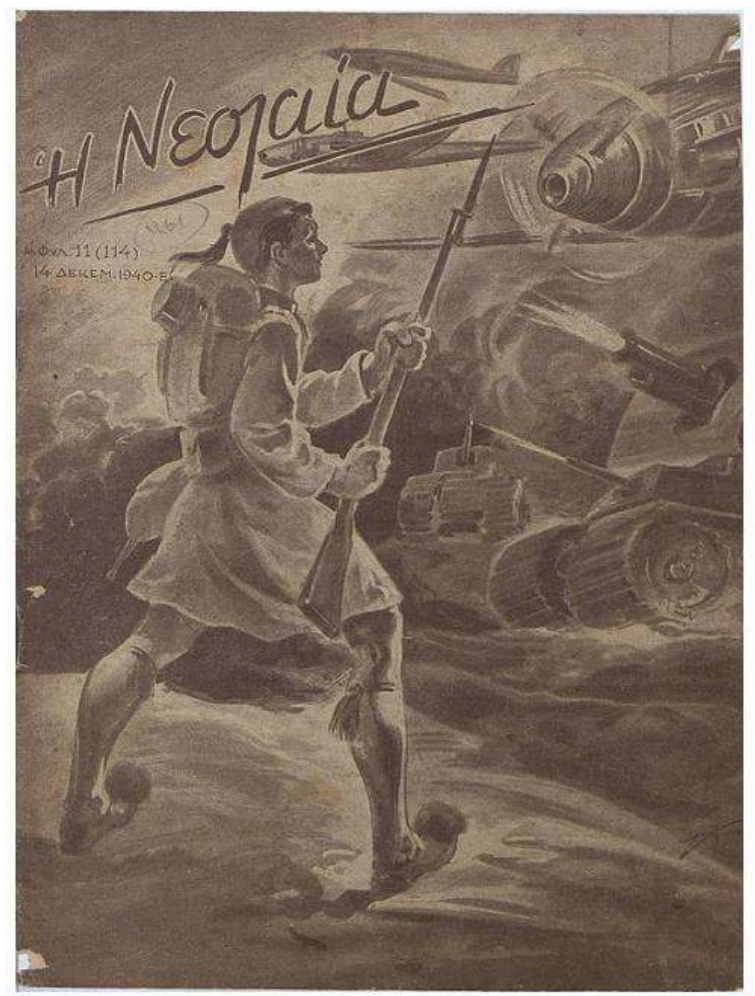

http://picasaweb.google.com/109214261579697097237/141940\#5454064313433530594 la pierre angulaire de la construction du Nous et de la résistance : «l'âme [...] transforme l'homme en héros, la pierre en Parthénon, la parole commune en Iliade» (N.25.01.1941, p. 1) et donne aux Grecs la capacité « depuis toujours d'affronter [...], la neige, le froid, la douleur » (N.15.03.1941, p.608) comme les soldats le font dans l'hiver 1940-41. Ces discours peignent le Grec armé d'une "cuirasse spirituelle invulnérable comme Achille» et d'une "conception chrétienne de l'histoire et de la vie» et qui "meure(nt) en Pallikare(s), pour l'Idée » (N. 9.11.1940, p. 161 et 15.02.1941, p. 510) et dessinent une nation délivrée des scories du matérialisme (N.18.01.1941, p. 409), et la supériorité d'un Nous habité par une force dont est dépourvu l'Autre. La guerre est donc une « initiation qu'accomplit une race tout entière" (N. 9.11.1940, p. 162) dont l'issue ne peut faire de doute, car, face à des "Italiens qui ne croient pas en leur combat contrairement à nous", " Nous les battrons, car ils sont Italiens et nous sommes Grecs » (N.11.01.1941, p. 401 et 16.11.1941, p. 196). Est-ce cette "âme grecque » qui permet à la modeste armée hellénique de repousser une armée italienne dépourvue de force morale?

\section{L'Italien, dernier avatar de l'Autre}

Si la Grèce doit faire face, en avril 1941, à l'invasion allemande qui, en quelques semaines, sera fatale au régime et à l'indépendance du pays, la propagande en direction de la jeunesse est alimentée essentiellement par les cinq mois de la guerre avec l'Italie, la destruction symbolique de l'Autre italien est indispensable.

Dans la revue datée du 11 novembre 1939, un an environ avant l'attaque italienne, phalangistes et pionniers sont appelés à se réjouir des discussions menées entre Grecs 
et Italiens pour maintenir la paix. Au prix d'acrobaties intellectuelles, l'article «T $\alpha$ $\varepsilon \lambda \lambda_{\eta v o i ̈ \tau \alpha} \lambda_{l \kappa \alpha \dot{\alpha})^{26}}$ tente de rassurer une jeunesse qui ne peut que s'inquiéter face aux événements. Le propos évoque «le flot des idées barbares» qui risque de submerger l'Europe, néanmoins, feignant d'ignorer la proximité idéologique et les liens diplomatiques et militaires, le rédacteur s'inscrit dans la logique d'apaisement choisie par Métaxas (et sans doute aussi soufflée par Londres). Dans cette perspective, histoires grecque et italienne sont lues comme les matrices de valeurs universelles menacées. L'Italien est certes Autre, mais égal dans l'héritage dont on le reconnaît porteur. Mais, à partir du 28 octobre 1940, le revirement est total et le magazine devient une tribune antiitalienne qui instrumentalise l'histoire pour peindre un Autre, antithèse du Grec.

Contrairement à la noblesse de la généalogie hellénique, les agresseurs italiens sont issus d'une lignée et d'« une race dégénérée » et corrompue (N. 14.12.1940, P. 288.). Des conquérants romains au peuple italien contemporain, « race de voleurs, [...] engeance de résidu de race ", en passant par les Génois et les Vénitiens, "rien d'autre qu'un peuple de brigands» (N. 7.12.1940 et 11.01.1941, p. 389), est affirmée une continuité malfaisante pour la Grèce et pour l'humanitée ${ }^{27}$. Ainsi, en peignant l'Italien ${ }^{28}$, se dessinent en creux les vertus et qualités grecques. Le discours peut aller jusqu'à la négation du statut d'homme (N.7.12.1940, p. 265), alors que les Grecs culminent au sommet d'une "surhumanité $»^{29}$. L'entreprise de propagande se manifeste aussi par la volonté d'une

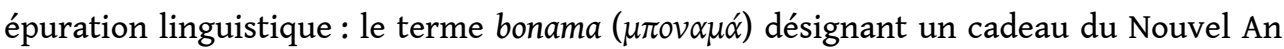
qui provient de l'italien bona mano se voit attribuer une généalogie grecque ${ }^{30}$ !

Spiritualité défaillante, absence d'âme chrétienne sont le produit des options religieuses au cours des âges. Triple est le péché commis : d'abord, sous l'apparence du Romain, l'Autre a persécuté le christianisme naissant quand, avec «les Caligula et Néron, [...] les viscères des chrétiens (étaient) dévorés par les Tigres». Puis Latins, Vénitiens, «il s'agit de catholiques (qui) contraignaient les Grecs à se convertir, chose que ne font même pas les Turcs » et "qui occup(ant) la Grèce [...] utilisaient l'assassinat ", ont trahi Byzance en 1204 et ont causé mille maux à l'orthodoxie. Enfin, dans le cadre fasciste, l'adoration d'un homme-dieu a encore corrompu les Italiens "ces nouveaux idolâtres [...] qui ont éteint depuis longtemps l'idée de Dieu dans leur âme " ${ }^{31}$.

20 La revue s'emploie inlassablement à réciter les crimes italiens au cours des âges et à les mettre en perspective avec les activités militaires du moment. La conquête romaine par Mummius et la destruction de Corinthe est ainsi associée au bombardement de cette même ville par ses "braves descendants» (N. 11.01.1941, p. 389), la destruction du Parthénon par le général vénitien ${ }^{32}$ Morosini fait écho au bombardement de Nicopolis et de son musée archéologique en mars $1941^{33}$. Italiens «qui ont toujours vomi leur bile aux Grecs [...] ont haï la Grèce du 4 août [...] ont haï la Grèce de 1912 et 1913, car le pays grandissait et réalisait ses rêves téméraires $"^{34}$, eux qui grâce "au fourbe plan du général Amélio " (N. 1.03.1941, p. 560) ont soumis le Dodécanèse. L'aboutissement naturel, sous la plume du Général en chef des armées grecques, Papagos, du funeste cortège des infamies italiennes étant "l'impérialisme effréné d'une grande Puissance» (N.2.11.1940, p. 133).

21 Si les crimes ont été perpétrés par un Autre au visage changeant, mais aux caractères invariants $\mathrm{s}^{35}$, les violences des Italiens de 1940 sont fréquemment associées à l'idéologie fasciste. Remarquable est l'irruption d'un terme que nulle part auparavant on ne rencontre dans la revue, que ce soit pour désigner le régime mussolinien ou lui associer le régime du 4 août. Après le 28 octobre 1940 et malgré une incontestable parenté 
idéologique et formelle que Métaxas affirme dans ses Pensées puisque «l'Italie a reconnu la similitude du régime avec le sien $»^{36}$, le régime propose une mobilisation contre la «mafia, [...] la camorra fasciste de Mussolini $»^{37}$, repoussoir, sans doute plus rhétorique qu'idéologique.

Ainsi l'ensemble de la propagande oppose-t-il civilisation et barbarie, lumineux idéalisme et obscur matérialisme, beauté et laideur, bien et mal, engagés dans un bras de fer séculaire qui a vu dès les origines les « fils de la Louve [...] construire le Colisée plutôt que des Parthénons et (en faire) des arènes de sang » (N. 14.12.1940, p. 290). L'Italien endosse à partir de l'automne 1940 le costume de l'Autre qui se dresse sur la route séculaire des Grecs, mais c'est aussi une figure mouvante et plurielle. En effet, l'obsession antiitalienne frontale peut laisser quelque espace à la dénonciation d'une autre altérité menaçante.

\section{Le danger « slavo-communiste » : le Bulgare en embuscade !}

Si les inquiétudes grecques se sont avérées fondées avec l'attaque italienne d'octobre 1940, une autre menace pèse. Comme l'Italie, la Bulgarie révisionniste cherche en effet à rebattre les cartes distribuées par les traités et menace la souveraineté hellénique en Macédoine orientale et en Thrace. En effet, depuis les traités de Bucarest (1913) et de Neuilly (1919), l'esprit de revanche anime de nombreux Bulgares et "la haine du Grec est ancrée dans les cœurs ${ }^{38}$ ». La méfiance qui s'avèrera fondée se traduit par les travaux de défense à la frontière gréco-bulgare - la ligne dite Métaxas $^{39}$-, elle s'exprime aussi dans la préparation psychologique de la jeunesse face à une agression qui semble probable.

Dans un conte de Noël publié en décembre 1940, les jeunes lecteurs doivent être édifiés par un dialogue mettant en scène des "villageois schismatiques bulgarophones" et un sergent Grec dans la Macédoine serbe. Le Bulgare se voit dénié son statut de chrétien

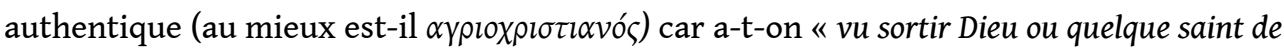
Bulgarie? » et, le Grec de se lancer dans une longue énumération de saints grecs alors qu'il met au défi son interlocuteur de trouver un exemple bulgare. Enfin après avoir affirmé que " tous les Grecs vont au paradis ", le soldat s'imagine en "gardien (de ce même) paradis que quelqu'un doit protéger afin que n'y pénètrent pas les Comitadjis ». S'opère ainsi une disqualification de l'Autre face à l'authentique chrétien (N.21.12.1940, p. 323). La proximité religieuse s'avère donc inopérante, et le Bulgare rejoint l'Italien dans une alliance objective du catholicisme romain et de l'orthodoxie schismatique.

Suspect et dangereux également, le Bulgare l'est en tant que Slave, ennemi séculaire du monde grec et la geste de Basile Bulgaroctone est présentée comme exemplaire puisque "l'empereur-soldat a arrêté l'ennemi aux frontières de l'hellénisme [..] hellénisme qui comme aujourd'hui était menacé par les Slaves» (N.2.11.1940, p. 138). Les Slaves représentent un danger permanent mettant en péril la survie de la civilisation grecque et, de ce point de vue, la propagande métaxiste emprunte manifestement la voie de Ion Dragoumis qui voyait dans ce voisin le danger premier pour l'hellénisme ${ }^{40}$. Le monde slave est aussi l'espace qu'une tradition juge étrangère à l'identité grecque. Les Slaves auraient une propension historique à la porosité au "miasme » communiste étranger à toute la tradition que prétend incarner le régime du 4 août, vision renforcée depuis les années 1920 par la tactique imposée par le Komintern qui vise à la création d'une Macédoine rouge ${ }^{41}$. 
26 Face à l'attaque hitlérienne contre la Yougoslavie et contre le royaume hellénique le 6 avril 1941, la jeunesse grecque se voit désigner un nouvel Autre jusqu'à lors étrangement absent des discours officiels. Les mêmes ressorts mis en œuvre contre l'Italien vont-ils être tendus contre l'Allemand?

\section{Introuvables Allemands, invisible nazisme?}

Depuis le début du conflit en Europe et malgré l'alliance anglo-hellénique, rares ont été les évocations de la guerre sur le continent, et à partir d'octobre 1940, la guerre grécoitalienne phagocyte la revue. L'Allemagne qui impose l'ordre nazi en Pologne, au Benelux et en France, n'apparait pas sur le « radar » de la propagande !

L'efficacité du blitzkrieg dans les Balkans ${ }^{42}$ au début du printemps 1941, conduit à une première constatation. La guerre contre l'Italie et ses cinq mois de durée avaient permis à la revue, à ses 23 numéros et à ses quelque 567 pages, de marteler la propagande. À l'inverse, seuls trois numéros constitués de 63 pages seront publiés entre les 12 et 26 avril 1941. D'un point de vue quantitatif s'impose donc cette première nécessaire distorsion entre le traitement effectif des deux ennemis.

La seconde observation repose sur un incontestable contraste dans le traitement des événements et la désignation du nouvel ennemi. Seuls deux articles assez brefs sont consacrés aux événements tragiques d'avril ; leurs titres ( $A u$ nouvel ennemi» et « La Grèce face au nouvel envahisseur») et contenus, dans une sobriété clinique, tranchent avec les envolées lyriques chargées de références historiques et la richesse sémantique de la prose anti-italienne. L'évocation de "l'ambassadeur allemand", des "troupes allemandes" ou de "l'envahisseur allemand» cohabite avec le rappel des méfaits du "pseudoCésar de Rome» (N. 12.04.1941, p. 698, 699). On ne trouvera pas d'« âne de Berlin» ni de "gangsters nazis»; le Führer et l'idéologie hitlérienne restent étonnamment ectoplasmiques...

Dans les deux numéros des 12 et 19 avril 1941, une page entière est consacrée à "l'armement dans le monde animal », une autre à " une mule victime du devoir », une demipage évoque le tombeau de Chistophe Colomb, et le poids de l'histoire et la puissance mobilisatrice du nouvel «OXl» (N. 19.04.1941, p. 740) ne reviennent que dans la page des petits Pionniers. Apparaît ici un contraste net avec les numéros de novembre 1940, riches de textes mobilisateurs et de photographies témoignant de l'enthousiasme populaire lors du déclenchement de la guerre.

31 Le 23 avril 1941, le général défaitiste Tsolakoglou ${ }^{43}$ signe une capitulation sans condition et Georges II accompagné de son gouvernement se réfugie en Crète et en

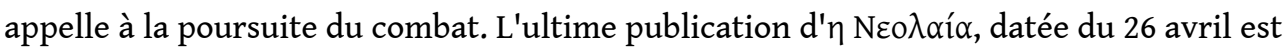
emblématique de la page qui se tourne : la revue compte environ deux fois moins de pages, mais alors qu'Antigone avait été sacrifiée sur l'autel de la guerre en novembre 1940, le récit des aventures de Robinson Crusoé se poursuit, un jeune tambour montre son courage dans une bataille sans ennemi identifié et un article puissamment mobilisateur (!) traite des «services météorologiques de l'armée ». La double page consacrée aux Pionniers a été drastiquement réduite et bien que la revue s'ouvre par la transcription des messages du monarque et de son nouveau Premier ministre, le fond historique et le souffle épique semblent épuisés. De même, si les illustrations des premières ou quatrièmes de couverture sont depuis octobre 1940 guerrières pour la plupart, le dernier numéro recycle une photo bien inoffensive des fêtes de l'EON au 
stade panathénaïque en 1938 : un jeune athlète armé de son javelot devant des gradins vides! Sur la quatrième de couverture, des hommes en costume traditionnel semblent prêts à esquisser un pas de danse plutôt qu'à combattre !

Plusieurs hypothèses peuvent être mobilisées afin d'éclairer ce constat dont le temps de réaction des rédacteurs et l'urgence de priorités jugées plus vitales. On peut aussi suggérer que le principe de réalité, - l'écrasante supériorité militaire allemande et la naissance d'un nouveau front-, a pu s'imposer après la «divine surprise » de l'hiver 1940 face à l'armée du Duce. La revue exprimerait ici une forme de fatalisme et son contenu afficherait implicitement cette "chronique d'une mort annoncée »: Léonidas, les Marathonomaques, les akrites byzantins et les héros de 1821 ont quasi disparu et le ton est bien souvent celui d'une oraison funèbre qui affirme certes l'immortalité de la Grèce, mais semble empreint d'une forme de résignation ( $\mathrm{N}$. 26.04.1941, p. 748, 756). Au-delà de ce fatalisme, peut-on envisager qu'une forme de défaitisme ait pu imprégner la revue ? L'attitude de l'une de ses principales rédactrices, Sitsa Karaïskaki, est pour le moins troublante. Rédigeant l'essentiel de la page des jeunes filles et divers articles, elle n'a de cesse, depuis octobre 1940, d'appeler à la mobilisation, or, face à l'attaque allemande et si elle fait sobrement référence en une occasion unique "aux ennemis", son discours manque d'ardeur dans la désignation du nouvel Autre. Acquise depuis longtemps déjà au national-socialisme, elle sacrifiera d'ailleurs son patriotisme à ses penchants idéologiques et quand les Allemands entreront à Athènes, elle choisira la voie de la collaboration ${ }^{44}$...

Alors, s'il est incontestable que les Grecs n'ont pas un contentieux historique avec les Allemands comparable à celui qui alimente les sentiments anti-italiens, s'il est avéré que les liens personnels de Métaxas avec l'Allemagne étaient étroits et que de nombreux ponts existaient entre son régime et l'Allemagne nazie ${ }^{45}$, les ambiguïtés et zones d'ombre demeurent nombreuses ${ }^{46}$ et on peut s'interroger sur la nature profonde du changement de ton de la revue au moment de l'attaque hitlérienne. Tandis que l'épopée séculaire grecque disparaît de la propagande, l'Allemagne nazie annexant l'histoire hellénique, fait de Léonidas un héros germanique-nordique, et de la Werhmacht, la colonne des nouveaux Trois-cents $!^{47}$ Comment faire de l'Allemand,

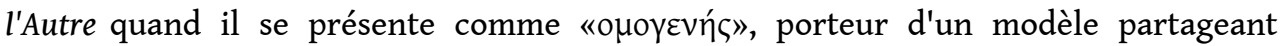
certaines valeurs avec le régime? L'Allemagne nazie se présente comme la puissance libératrice d'une « race » qui a été corrompue, et celle qui mettra fin à la «schizophrénie raciale et spirituelle des Grecs, tiraillés entre leurs origines nordiques et les ferments allogènes [...] qui se sont insinués dans leur sang " ${ }^{48}$, les " germes " portés par les " untermenschen " slaves et juifs et les «miasmes » asiatiques. L'occupation ottomane constitue donc l'un des facteurs de cette mixtion tragique des races pour les nazis; or dans le contexte de la guerre, le régime du 4 août va façonner pour la jeunesse une image du Turc pouvant à la fois ménager la susceptibilité du voisin oriental et participer de la destruction symbolique de l'Autre.

\section{Turcophilie et aseptisation du passé au service des intérêts géopolitiques}

Dans un pays où la construction et l'identité nationales ont été façonnées dans les siècles douloureux de la Turkokratia, les heures du combat révolutionnaire puis des guerres gréco-turques et de la Catastrophe de 1922, la figure du Turc s'impose au 
sommet de l'altérité. Or, la vision grecque du Turc (en amont et en aval de la période métaxienne d'ailleurs) est négociée selon les nécessités du temps.

Avant l'accession au pouvoir de Métaxas en 1936, la Grèce de Vénizélos puis de Tsaldaris avait cultivé un rapprochement avec le voisin turc en concluant des accords bilatéraux en 1930, puis 1933, complétés par le pacte balkanique en 1934. Le régime du 4 août ne rompt pas avec cette orientation diplomatique et resserre les liens dans le contexte de la montée des périls en Europe et de la prégnance de la menace bulgare ${ }^{49}$. Métaxas qui se rend en visite officielle à Ankara en 1938, signe un nouveau pacte avec le voisin oriental et resserre l'Entente balkanique. Au lendemain de la disparition d'Atatürk, en novembre 1938, la revue souligne son « œuvre titanesque dans la réalisation de l'amitié gréco-turque" entre deux peuples qui ont en commun les mêmes "vertus humaines» (N.25.11.1938, p. 246); de même, le cahier photographique de l'hebdomadaire, pavoisé aux couleurs grecques et turques, montre l'enthousiasme des phalangistes et des Athéniens lors de la visite du ministre turc des Affaires étrangères

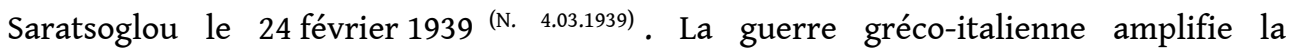
réévaluation de l'altérité turque et sa présentation à la jeunesse ${ }^{50}$, le récit des relations gréco-ottomano-turques sera infléchi par la dénonciation de l'altérité absolue et essentialisée de l'Italien ou du Bulgare.

Les jeunes Grecs sont appelés à transférer les sentiments hostiles qu'ils pourraient avoir à l'encontre du Turc. On passe sous silence de nombreux aspects de la conquête, de l'occupation ottomanes et des conflits qui ont opposé Grecs et Turcs et l'on rappelle que "les Péloponésiens préféraient la servitude au Sultan [...], car les Vénitiens saisissaient leurs biens, [...] les soldats dormaient dans leur maison [...] et on les obligeait à se convertir (au catholicisme) chose que ne font pas les Turcs eux-mêmes» (11.01.1941, p. 389). L'antagonisme religieux entre orthodoxie et islam est supplanté par les persécutions « italo-catholiques ». Dans l'art de la guerre, aux manœuvres perfides, à la ruse, à la couardise italiennes ainsi qu'aux bombardements barbares de populations civiles, la propagande oppose des "Turcs adversaires généreux et chevaleresques", "brave(s) et opiniâtre(s)»(N.03.1941, p. 556). Cette même tonalité s'impose dans le champ de l'histoire diplomatique, car les terres grecques irrédimées ont échappé au rattachement à la mère patrie en raison des manœuvres italiennes puisqu'«Au Congrès de Berlin, même les Turcs ne nous ont pas paru aussi ennemis que les Italiens, surtout s'agissant de l'Épire" (N. 30.11.1940, p. 244). L'histoire des relations gréco-turques est donc expurgée et les Turcs se voient parés d'une humanité qui rapproche, là où la barbarie historique exclut l'Italien de la communauté des hommes. La guerre propulse les Turcs vers les sphères proches du nous, car " (ils) sont aujourd'hui un sûr, sincère et brave allié " (N. 28.12.1940, p. 337). Ce Nous qui, dans le contexte du conflit et d'une bipolarisation planétaire trouve des élargissements dans un monde anglo-saxon dont pourtant Métaxas et l'idéologie du 4 août réprouvent largement le modèle.

\section{Avec Britanniques et Américains : un mariage de raison?}

37 Le régime du 4 août se trouve confronté à une nécessaire gymnastique intellectuelle et idéologique pour légitimer aux yeux de la jeunesse l'alliance britannique et le soutien américain.

Certes, dans les années qui précèdent la guerre, la revue a multiplié les articles dénonçant les menaces mortelles que constituent marxisme et communisme, mais la 
propagande s'est aussi attachée à démonter le modèle démocratique et libéral et son corollaire mortifère qu'est l'individualisme (N. 25.11.1938, p. 227) comme les effets délétères $\mathrm{du}$ capitalisme industriel et financier et son culte de la réussite matérielle. De très nombreux articles mettent en lumière la «vraie liberté » définie comme "liberté disciplinée » et rejettent, comme non helléniques, la liberté politique et de la démocratie parlementaire. Le destin et l'intérêt de la communauté, de l'ensemble national, s'imposent, mais le culte de la production matérielle est présenté comme une menace pesant sur l'idéalisme hellénique, et les pratiques du capitalisme financier "ploutocratique» sont opposées aux vertus cardinales du labeur agricole. Jamais cependant ne sont mis au pilori Britanniques et Américains, même si dans ses écrits personnels, Métaxas est plus explicite : "Cette démocratie, democracy (en anglais dans le texte), est l'enfant du capitalisme. C'est l'organe par lequel le capitalisme domine les masses populaires [...] (pour) faire des hommes ses esclaves, esclaves qui pensent être libres » et plus loin: "Les Anglais veulent détruire, disent-ils, le nazisme. Mais en fait ils veulent dire l'antiparlementarisme. Ils veulent imposer partout le système libéral vétuste $»^{51}$. Malgré ces préventions, les menaces confirment la realpolitik ${ }^{52}$ qui subordonne l'idéologie aux nécessités géopolitiques et conduit la Grèce dans le camp des démocraties occidentales.

Avec les invasions italienne puis allemande, l'heure est venue de mettre en valeur le combat commun et la fraternité qui unissent le peuple grec et les nations britannique et américaine. Au-delà des images ou des mots qui traduisent cette alliance, comme la photographie des "aviateurs anglais objets de chaleureuses manifestations du peuple athénien" (N. 9.11.1940) au lendemain de l'entrée en guerre, ou la publication du message de Winston Churchill à Ioannis Métaxas dans lequel il affirme « La façon dont le peuple grec a affronté les dangers et les provocations depuis quelques mois a suscité l'admiration du peuple britannique» (N. 9.11.1940, p. 191), la mobilisation de l'histoire, là aussi, alimente la propagande.

Elle s'articule autour d'un point de vue hellénocentré: le monde anglo-américain (portion de la civilisation occidentale) a contracté une dette envers la Grèce qui, aux Thermopyles ou à Salamine, faisant rempart aux « hordes barbares » a sauvé l'Europe de l'engloutissement asiatique (N. 9.11.1940, p.191) et a ainsi rendu possible le développement de «la » civilisation sur le continent et outre-Atlantique. Toutefois, la propagande présente aussi une Grèce débitrice à l'égard du philhellénisme historique de ses alliés à travers la figure symbolique de Byron qui, comme Eschyle avant lui, engagé par le verbe, mais aussi les armes à la main à Marathon, a fait don à la Grèce de sa vie. De même, la revue accueille avec enthousiasme la loi prêt-bail américaine et salue «l'Amérique [...] le premier pays qui a reconnu la Grèce comme État» (N.22.03.1941, p.626). Enfin, elle mobilise son lectorat en convoquant des témoignages britannique et américain, comme une prière diffusée dans des milliers d'églises britanniques, prière pour une Grèce qui a "dispensé au monde entier l'esprit, la sagesse et le bien" (N.1.03.1941, p. 559).

41 Voici donc l'histoire mise au service d'une alliance orthodoxo-anglicane! Le peuple grec, grâce à des vertus qui lui sont propres, force l'admiration britannique aujourd'hui comme hier. Les mots de l'amiral anglais Hamilton en 1822 qui affirme que «Ces hommes (les Grecs) se libéreront ! ...], car avec ces vertus (frugalité, austérité, patience), ils sont au-dessus de tous les peuples aujourd'hui» (N.5.04.1941, p. 693) renvoient aux sacrifices consentis dans l'hiver épirote. La continuité historique et épique de l'ethnos hellénique alimente également les discours des alliés occidentaux et l'ambassadeur américain 
Lincoln Mac Veagh tient des propos publiés dans la revue qui font de lui un promoteur objectif du régime, car le "dévouement (des Grecs) est toujours concentré vers la famille, la religion et la Patrie [...] trois choses indissociables " ${ }^{53}$, ce triptyque (auquel est ajouté le Roi) constituant la profession de foi du régime depuis sa naissance. Mais, tout en invoquant Justice, Civilisation comme valeurs communes à la Grèce et à ses alliés, les discours omettent toute référence au modèle démocratique dont la défense est pourtant l'un des moteurs de la guerre menée par les Britanniques et les Américains !

$\mathrm{Au}$ terme de ce premier temps, on a pu mesurer comment l'histoire instrumentalisée par le régime du 4 août alimente la mobilisation de la jeunesse dans le combat, mais aussi comment la nouvelle donne géopolitique, diplomatique et militaire conduit à des redéfinitions ou révisions dans la désignation du Nous et de l'Autre. La grille de lecture du conflit dispensée aux jeunes Grecs doit se traduire dans leur comportement: comment les éonistes doivent-ils agir pour se conformer à un glorieux modèle en faisant leurs ces nouvelles altérités?

\section{L'EON engagée dans le combat}

L'histoire telle qu'elle est instrumentalisée par le régime sert bien évidemment à l'armement idéologique et psychologique de la jeunesse, mais, au-delà de ce cadre, la mobilisation exige des formes différentes et spécifiques en fonction de l'âge et du sexe des membres de l'organisation de jeunesse. Édifié par les leçons du passé, dans les temps tragiques du conflit, chacun à sa place se voit assigner un rôle et fait l'objet d'une injonction à agir et à se sacrifier dans la guerre totale ${ }^{54}$.

\section{Les nouveaux Trois-cents : la jeunesse combattante}

Dans l'ultime numéro de la revue avant le déclenchement de la guerre, un montage photographique montre de jeunes Phalangistes armés de fusils en bois entre les images de fantassins et de marins. Or, après le 28 octobre, parmi les jeunes gens mobilisés obligatoirement à partir de l'âge de 21 ans (et 20 dans le cadre du volontariat), nombreux sont les membres de l'EON qui recrutait jusqu'à l'âge de 25 ans. Ayant troqué l'uniforme du Phalangiste pour celui du Tsolias, quittant les exercices militaires dans les camps d'été pour le terrible hiver albanais, ils affrontent l'ennemi. La revue fait d'eux des modèles : trempés dans le passé héroïque, ils écrivent à leur tour l'histoire glorieuse de la Grèce et dessinent son futur.

L'exaltation du sacrifice des éonistes morts au combat se traduit dans trois numéros par une rubrique nécrologique dédiée aux «Enfants de l'EON tombés pour la Patrie ${ }^{55}$. Douze jeunes hommes «tombés héroïquement [...] dans l'exercice de (leur) devoir [...] pour l'indépendance grecque » dont les photographies accompagnent les noms, grades, lieux et dates du décès, apparaissent ainsi comme les dignes héritiers de tous ceux dont le sacrifice a jalonné « l'éternel combat » grec. Ils sont appelés à les rejoindre dans " une mémoire sacrée éternelle qui nourrira les générations futures de la jeunesse " et "la Gloire grecque inscrit leurs noms sur les pages immortelles de l'histoire de la Grèce» (N. 8.03.1941, p. 581). Les mânes des aïeux couronnent la nouvelle génération combattante ${ }^{56}$ ! Cette communion de tout un peuple avait déjà connu ses prémisses avec l'instauration

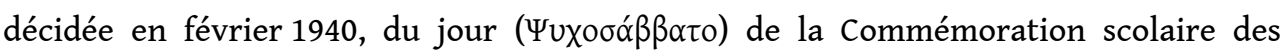
morts à la guerre qui culminait dans une cérémonie devant le tombeau du Soldat 
inconnu. Les portraits des jeunes gens et leurs épitaphes deviennent autant de " tombeaux de papier » aussi glorieux que le marbre de Syntagma! Ce consentement au sacrifice est présenté comme le fruit d'une conscience historique propre aux Grecs, car si le soldat «peut confondre Périclès et Thémistocle, cela n'a aucune importance. L'histoire de la Grèce, il ne la possède pas comme une froide connaissance. Il l'a en lui comme un sens, une substance, comme une réalité vivante » (N.15.02.1941, p. 510). Ainsi, la fiction historique édifiante ${ }^{57}$ remplit-elle les pages de la revue.

La vision de l'histoire et l'instrumentalisation qui en est faite s'inscrivent dans le concept de la "Troisième civilisation hellénique $»^{58}$ qu'affirme forger le régime et les vertus du jeune soldat nourri des « leçons de l'histoire » de l'EON, dessinent un modèle de comportement qu'on peut qualifier de spartiato-orthodoxe. L'héritage de l'éducation et du mode de vie, de la discipline et du sens du sacrifice spartiates a façonné l'héroïsme des jeunes combattants dont les Thermopyles sont les montagnes du Pinde. Déjà avant le début du conflit, la revue présente dans une rubrique régulière

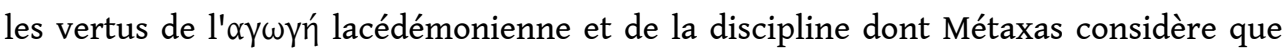
"présente dans les temps antiques [... ] (elle) a manqué durant les cent années de notre liberté ${ }{ }^{59}$. Ignorant les effets émollients du confort comme ses illustres ancêtres laconiens, la frugalité fait du jeune Grec (N.11.01.1941, p. 401) un combattant qui peut se battre avec moins d'aliments». Les jeunes comme "Léonidas [...] préféreront la certitude d'une mort glorieuse à une fuite honteuse" (N.15.02.1941, p. 509), ceux qui tombent au champ d'honneur sont « revenus » sur leur bouclier ${ }^{60}$ ! Mais la « panoplie spirituelle » qui doit équiper le jeune soldat ne saurait être complète sans la foi chrétienne qu'incarne un autre credo du régime : « Foi, combat, victoire » établit clairement qu'aux « soldats du Christ » tombés dans la lutte sacrée est promis le triomphe ${ }^{61}$. Éternels ils seront dans la mémoire des hommes et dans le royaume des cieux. Peut-on alors considérer que derrière les sacrifices expliquant les victoires contre les Italiens, la fougue guerrière s'est enracinée dans «l'émotion nationale (qui) fusionne avec l'émotion religieuse ${ }^{62}$ ».

Le devoir militaire qui incombe aux éonistes mobilisés constitue le suprême don de soi et illustre tragiquement la matérialisation d'une conviction idéologique fondamentale qui considère bien avant le début de la guerre que "le modèle de la Jeunesse est le soldat " ${ }^{63}$. Mais des centaines de milliers d'autres membres de l'organisation de jeunesse se voient sommés de participer à l'effort de guerre.

\section{La mobilisation symbolique et matérielle de la jeunesse}

48 L'enfant-soldat constitue un autre modèle qui se perpétuerait dans l'histoire des Grecs et que convoque la propagande à travers les exemples spartiate et souliote pour souligner la continuité à travers les âges de la bravoure et du sens du sacrifice de très jeunes garçons. Ainsi en 1940, «les enfants de l'Épire, dont beaucoup, étaient membres de l'EON", et qui participent au combat «en prenant des armes, en jetant des pierres ou des grenades ", sont un visage récurrent de la propagande, «l'histoire se répète à nouveau » (N. 30.11.1940, p. 251).Néanmoins, la participation réelle au coup de feu contre l'ennemi est rarissime, c'est virtuellement et symboliquement que les plus jeunes éonistes vont vivre cette expérience. La propagande peut nourrir l'imaginaire de petits héros d'exploits militaires, mais le sacrifice a lieu ailleurs et si le sang n'est pas versé sur le champ de bataille, leur devoir est de donner aux services médicaux ce sang qui 
provient des "profondeurs sacrées des siècles, de la grande et immortelle âme grecque ( $\mathrm{N}$. 22.02.1941, p. 531).

Métaxas affirme que « chaque membre de l'EON qui n'est pas mobilisé au combat est un soldat de l'intérieur " $"$. Mobilisation onirique nourrie de l'exemplarité des figures mythiques du passé. Mobilisation psychologique collective par la fièvre des serments enthousiastes: "Mourrons " comme les "pères l'ont fait chaque fois que la Liberté et le sol sacré de la Grèce étaient menacés $»^{65}$. Aucune des pratiques sociales ne peut échapper au contexte du conflit, une guerre que l'on peut mimer, comme l'illustre la représentation des jeunes Pionniers en arme battant la campagne dans chacune des pages qui leur est spécifiquement consacrée (o $\kappa \eta ́ \pi \circ \varsigma \tau \omega \nu \Sigma \kappa \alpha \pi \alpha v \varepsilon ́ \omega v \mu \alpha \varsigma$ ). La vraie guerre, elle, se gagnera par un moral d'acier des combattants et les plus jeunes ont la responsabilité de contribuer à le forger par des pratiques altruistes, la camaraderie et la solidarité que vante la propagande ${ }^{66}$.

Dans une guerre qui s'enfonce dans la durée et dans les terribles conditions climatiques $\mathrm{du}$ front albanais, les aspects matériels prennent une importance capitale et, dans ce domaine aussi, la pression sur la jeunesse est forte. Dès le début du mois de novembre 1940, est créée une branche de l'EON qui a vocation à soutenir matériellement les soldats et leur famille et à participer à la solidité du moral sur le front. Le Service central de Solidarité avec le Front (KYAM) dont la fondation est annoncée dans la revue affirme que "Tous (ont) l'obligation de participer au combat, car la plus petite contribution participera à la victoire finale $~^{67}$. Illustrant la nécessaire solidarité nationale, et mettant en exergue le fondement chrétien du don ; argent, vêtements, nourriture, cigarettes, ou jouets pour les enfants sont demandés à la générosité des phalangistes et des Pionniers qui se transforment en agents de collecte ${ }^{68}$. Ces pratiques ont été préparées avant la guerre par l'exemplarité de l'évergétisme des grands bienfaiteurs de la nation.

51 La Jeunesse nationale est donc mobilisée à des "postes de combat » différenciés selon l'âge des membres de l'organisation sur le front, mais aussi à l'arrière, dans une guerre totale exigeant son «matériau » humain, psychologique et matériel. Les filles quant à elles occupent une place spécifique dans les discours de propagande.

\section{Filles et jeunes femmes : dociles Iphigénie, farouches Spartiates ?}

L'exploration de la propagande doit s'orienter à la fois vers les messages adressés spécifiquement à la portion féminine de l'EON mais aussi au repérage des discours sur la place et au rôle des femmes qui s'adressent à l'ensemble de la société.

53 L'idéologisation et la politisation du rôle des femmes et des filles constituent une des lignes directrices du régime. Tentation ou tentative totalitaire et obsession de la palingénésie provoquent leur "irruption » dans la sphère publique. Renaissance ou régénération nationales occupent une place primordiale et les jeunes filles constituent à la fois un "matériau " que le régime doit sculpter et un vecteur essentiel dans la réalisation de ses objectifs. Or, la thématique de la décadence de la société grecque est centrale dans l'idéologie du 4 août : féminité exacerbée, frivolité et activisme féministe remettant en cause l'« idéal féminin » et le système patriarcal, constituent les maux qui mineraient l'archétype séculaire. La propagande dénonce donc les pratiques corruptrices qui menacent d'éteindre «les lumineuses valeurs féminines grecques [...] (quand on) [...] demand(e) à des Phryné, des Aspasie de remplacer des Nausicaa et des Pénélope.[...] Car c'est une grande catastrophe quand dans un peuple on ignore la Femme-mère 
et qu'on reconnaît seulement la Femme-hétaïre ${ }^{69}$. La propagande cultive donc la promotion de la femme au foyer, épouse et maman souveraine en son "royaume » domestique tandis qu'à l'école et dans les camps d'été les jeunes filles reçoivent un enseignement spécifique pour la «femme au foyer $»^{70}$. Parallèlement ces centaines de milliers de fillettes et de jeunes filles enrôlées dans l'EON, vêtues des mêmes uniformes bleu et blanc que les garçons (sauf la jupe), sont amenées à sortir du "gynécée ", à recevoir des leçons idéologiques, à se mêler à leurs homologues masculins, à défiler au pas avec eux et à se livrer à des activités athlétiques ${ }^{71}$ (dans lesquelles les corps sont parfois assez dénudés) et à certains exercices militaires (entraînement au tir). Incontestablement, ces activités et cette mixité peu conventionnelles en Grèce ont pu brouiller l'image de la répartition sexuée des tâches et revêtir un caractère émancipateur ${ }^{72}$, mais elles ne doivent pas en cacher les finalités (forger l'obéissance, la discipline, la dévotion au régime, préparer des corps sains à la maternité et préparer à la guerre). Par conséquent, si les jeunes filles sortent du foyer familial et si la répartition traditionnelle des rôles est parfois transgressée par certaines activités extérieures, le discours idéologique les renvoie vers "l'intérieur " dans de strictes formes conservatrices. Quel est le modèle qui s'impose à une fille à qui l'on propose d'une part, l'image de jeunes femmes en maillot de bain se livrant à des activités sportives sur la plage et, d'autre part, celle de la femme vêtue traditionnellement, gardienne du foyer barattant le beurre? Doit-on y voir des injonctions contradictoires dans un corpus idéologique peu cohérent ou des tensions diverses au sein de l'appareil de propagande? Le régime ne libérerait-il provisoirement les jeunes filles de l'emprise masculine, patriarcale et domestique que pour mieux y renfermer les femmes? Quand Sitsa Karaïskaki affirme à la jeune lectrice : " [...] ni ta grand-mère, ni ta mère n'ont vu de tels jours historiques. Elle était enfermée à la maison sans qu'il se trouve personne pour lui faire connaitre sa grande mission [...] Jeune fille toi aussi tu travailleras à la reconstruction d'une Nation" (N. 26.11.1940, p. 240) exprime-t-elle le passage d'une soumission à l'univers domestique à celui de la communauté nationale enrégimentée?

54 Avec la guerre, si la thématique du sacrifice imprègne l'ensemble du discours, quelles doivent en être les formes? La combattante au cœur de l'action militaire peut constituer pour les lectrices un modèle inspiré de ces femmes «crétoises, souliotes (qui) prennent les armes et (prouvent que) [...] la Grecque n'a pas changé, brave, héroïque au travers des siècles ${ }^{73}$. Mais cette possibilité renvoie à des circonstances exceptionnelles comme à Sparte où « le Roi combattait dans une autre guerre, [...] en 1821 quand les ennemis étaient beaucoup plus nombreux» (N.15 03 1941, p. 616), et la "normalité » explique que «La Grecque n'a pas pris les armes, car son éducation et sa tradition ne la veulent pas rude et virile» et "car le mythe des Amazones [...] a été et reste étranger à l'âme grecque » (N. 8.03.1941, p. 592). Antagonique aussi l'exaltation des femmes de l'Épire, déblayant la neige sur les routes, ravitaillant en munitions et nourriture les combattants, présentés comme d' « antiques Spartiates (qui) revivent dans le Pinde » ${ }^{74}$ et l'affirmation que "le Grec ne veut pas voir sa femme, sa sœur [...] marcher dans les montagnes, souffrir [...] être sur les lieux du combat [...] il veut la sentir protégée à la maison» (N. 8.03.1941, p. 592). L'histoire peut donc attester simultanément de la possibilité d'une frontière mouvante entre les sexes, mais aussi d'invariants qui ne sauraient être transgressés ${ }^{75}$. Seule la phalangiste infirmière pourrait incarner une forme de résolution des contradictions « dehorsdedans ».

Il semble que la guerre renforce largement la distribution conventionnelle des rôles. "Soldat de l'arrière ", " Notre propre front ", " Notre guerre ", le vocabulaire est guerrier, 
mais les formes de la mobilisation renvoient pour l'essentiel au foyer. En tant que " soldat spirituel ", la jeune fille, armée des vertus de son ascendance spartiate ${ }^{76}$ et de sa foi chrétienne, doit entretenir le moral de «son » soldat (père, frère, membre de la famille) et le galvaniser "certain de l'esprit qui souffle à la maison». Mais la solidarité nationale exige aussi que la jeune fille ou la fillette s'extraient du strict milieu familial.

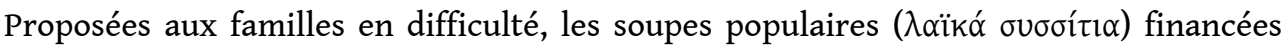
par l'État et dans lesquelles la phalangiste sert des repas constituent une autre forme du devoir de celle "qui n'est qu'une unité de l'ensemble» (N. 5.04.1941, p. 688). De même l'accueil à sa table de l'enfant pauvre d'un soldat mobilisé doit être un devoir sacré et le tricot devient l'activité patriotique par excellence : armée de «(Son) aiguille à tricoter (qui) est (sa) lance " ${ }^{77}$, la jeune fille est exhortée dans de multiples messages à confectionner chaussettes et gants qui «protègeront nos héros des engelures» (N. 12.04.1941, p. 716). Vouées à des activités conventionnelles érigées en œuvre de salut national, les jeunes femmes doivent aussi se préparer à remplir leur devoir maternel et former les «mamans du futur » qui feront du " petit d'aujourd'hui [...] le valeureux Grec de notre Patrie» (N. 14.12.1940, p. 302 et 1.03.1941, p. 568). La «nationalisation de l'utérus $»^{78}$, amorcée avant la guerre, ajoute à la contribution à la Régénération, la participation à la survie de l'indépendance nationale, car «la Maman [...] comme les antiques Spartiates, les anciennes Souliotes escorte avec son patriotisme et son affection les mobilisés » (N. 2.11.1940, p. 141).

L'histoire, telle que le régime l'instrumentalise, offre donc à la jeune membre de l'EON une palette complexe de modèles dont il est difficile d'établir la cohérence. Est-ce la figure sacrificielle d'Antigone ou d'Iphigénie, l'inflexibilité de la sœur ou de la mère spartiate, l'héroïsme de la combattante de 1821 qui doivent l'inspirer ? ${ }^{79}$ Il semble néanmoins que, si les années 1936 à 1940 avaient pu voir se redessiner les contours des conventions sexuées avec des jeunes filles en partie soustraites au milieu familial, le contexte de la guerre conduit à un retour à des formes plus traditionnelles dans la répartition des rôles. Les jeunes éonistes victimes de la guerre, contrairement à leurs camarades masculins, n'auront pas leur «monument » mémoriel dans la revue! Mais les occupations allemande, italienne et bulgare jetteront au cœur du combat contre l'oppression, avec des mots d'ordre idéologiques et politiques radicalement différents, des milliers de jeunes filles de l'EPON ${ }^{80}$ !

La guerre a renforcé l'enrégimentement de la jeunesse et l'exigence du sacrifice de chacun. Des plus petits bambins jusqu'aux jeunes adultes, tous ont une mission à remplir et la lourde tâche de se montrer dignes de l'héritage illustrant ainsi les mots de Métaxas: "nous ne nous appartenons pas à nous même, mais nous appartenons à la Patrie grecque $»^{81}$. Le conflit renforce donc l'un des piliers de l'idéologie du régime: la soumission du destin individuel à celui de la communauté.

\section{Le passé au service du régime : la guerre comme « opportunité »}

«Je suis persuadé que cette guerre [...] donnera au Peuple grec une nouvelle puissance et lui permettra d'ouvrir plus grand ses ailes [...]. Ainsi mon rêve [...], la création d'une troisième civilisation [...] deviendra réalité $\aleph^{82}$. Ces propos de Métaxas au début de l'année 1941 témoignent de la conviction chez le dictateur que le conflit sera un accélérateur de l'histoire. Bien sûr la défense de la Patrie imprègne l'ensemble des 
discours, mais si certains ${ }^{83} \mathrm{y}$ voient la relégation des ambitions démiurgiques du 4 août, il semble que la guerre a pu fournir un tremplin aux idées de "Renaissance » de la Grèce, car « l'esprit de la guerre devra nourrir la paix » (N. 11.01.1941, p. 388). En effet, alors que le combat contre les «ennemis intérieurs» constitue l'axe central de la propagande dans la revue avant la guerre, la mobilisation contre l'ennemi étranger phagocyte le contenu depuis octobre 1940. Néanmoins, l'aspiration à forger une société monolithique en sort renforcée.

\section{Réaliser l'unité nationale et la caporalisation de la société}

59 Si les longs et nombreux articles théoriques dénonçant «l'esprit de classe » et le " péril communiste» disparaissent, un court article traduit l'obsession de l'unité et la réintégration possible des marxistes repentis dans la communauté nationale. L'apostasie de Sérapheim Maximou est donnée en exemple de "rédemption »: «Il ne croit plus » et «[...] (se) trouve en opposition théorique avec le marxisme, [...] désapprouve l'application de cette théorie telle qu'elle est appliquée en Russie [...] " ${ }^{84}$. À cette " foi » perdue doit se substituer le "Je crois en la "race» grecque [...] en la Grèce unie» (N. 22.01.1941, p. 544.) et la vision d'une société travaillée par la lutte des classes et la division nationale se voit encore davantage disqualifiée. Métaxas ne pourrait être plus explicite quand il affirme que "[...] la guerre et la solidarité [...] détruiront, si elles existent encore les dernières barrières (de classe) [...] $\aleph^{85}$. Parallèlement au communisme, et constituant avec celui-ci la « double tyrannie » dont aurait souffert la Grèce contemporaine, les « maux » inhérents à la démocratie et au parlementarisme ${ }^{86}$ que le régime a toujours dénoncés, pourront être définitivement éradiqués par la guerre. La communauté nationale,

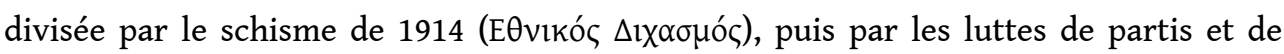
personnes dans les années vingt et trente se voit ressoudée par l'adhésion à la " catéchèse " métaxiste et le soutien au régime d'un journaliste ancien adversaire du général. C'est en puisant dans le fond mythicohistorique de l'idéologie du régime que Christos Andréou Manessis répond "présent(s)» comme autrefois «[...] Akrites et Pallikares [...]» et fait allégeance au dictateur et à "son cuvre» de construction de "palais sur la nouvelle Acropole»(N. 30.11.1940, p. 242.). La revue accompagne sa profession de foi d'un appendice édifiant visant à démontrer l'inanité des luttes partisanes. Devant le modèle spartiate, la démocratie athénienne disparaît purement et simplement de l'histoire ${ }^{87}$. La propagande promeut le projet d'une société lisse, sans aspérités et le jeune lecteur est mis en garde : les leçons de l'histoire sont sans appel, la Grèce a été brisée par ses ennemis quand elle était divisée ${ }^{88}$. Sans aucun doute, pour les tenants du régime, ce consensus national devrait-il perdurer après la guerre.

Avec la guerre, le régime trouve aussi l'occasion de renforcer l'unité nationale autour des valeurs centrales que sont pour lui l'obéissance et la discipline. Le conflit dans lequel est plongé le pays exige que des formes de "déviance" liées à des comportements individualistes soient annihilées. D'une part, "L'amour de la Patrie et l'obéissance à ses lois" sont un devoir premier du Grec et Phalangistes et Pionniers devront s'inspirer des exemples des Miltiade, Alcibiade, Aristide, Thémistocle et Socrate qui ont préféré la mort à la trahison de leur soumission à la loi (N. 9.11.1940, p. 183). S'exprime aussi l'exigence d'une discipline dont selon Métaxas, "l'absence a été la cause de nos grandes infortunes» (N. 25.01.1941, p. 434). Il faut donc être pleinement hellène par "la discipline, l'ordre - choses auxquelles le romios n'adhère pas avec empressement $»^{89}$. Ainsi, puisant aux sources d'un passé soigneusement composé, le 
régime trouve dans la guerre les conditions favorables à l'accomplissement d'une unité sans faille et de l'enrégimentement de la société qui devront continuer à prévaloir après le conflit.

61 Le peuple grec ne formerait ainsi plus qu'un, animé par la "flamme sacrée " et abandonnant «[...] doute, scepticisme, crainte [...] tous engendrés par l'esprit individualiste et matérialiste » pour devenir « [...] êtres humains authentiques, hommes authentiques, c'est-à-dire Grecs authentiques » (N. 9.11.1940, p. 163). Exaltation de la communauté nationale, rejet de l'« ineptie » démocratique et de son «vieux personnel politique $»^{90}$ disqualifié, associés à un anticommunisme viscéral dessinent une vision sinon fasciste, du moins fascisante, il ne s'agit sans doute pas seulement d'une " union sacrée " face au péril du moment, mais d'un projet à dimension totalitaire visant à forger société et homme nouveaux.

\section{Consolider le culte du chef}

Alors que l'apparence de Ioannis Métaxas, selon le ministre italien de l'Instruction Giuseppe Bottai «ne révèle en rien le militaire, en rien le dictateur [...], mais au contraire une ressemblance avec un instituteur", que Laird Archer le perçoit comme "un magistrat provincial âgé $\|^{91}$, la guerre offre à la propagande l'opportunité de renforcer l'autorité d'un dirigeant bien peu charismatique.

L'idée d'un homme "élu parmi les élus $»^{92}$ se construit par la combinaison d'une filiation familiale et d'une filiation "phylétique ». C'est la «Coïncidence heureuse »: Métaxas " $a$ vu le jour au même endroit qu'Ulysse "!, c'est aussi l'histoire d'une famille dont les origines et les migrations, de la Macédoine à Constantinople en passant par les îles Ioniennes, "avec un mélange de sang de toutes les régions", dessinent une parfaite "géographie nationale ». L'évocation du sang versé par les Métaxas dans les combats nationaux et la mention d'un certain César Métaxas, fusillé par les Vénitiens, valent brevet de patriotisme et dessinent une généalogie familiale héroïque qui permet au général de posséder d'admirables "vertus héréditaires». C'est aussi un héritage «national» dont est porteur le dirigeant. Personnalité cultivée avide de «tout apprendre", combattant national ayant "participé à toutes les phases de l'effort pour réaliser (le) rêve d'un rétablissement plus complet de la nation", acteur politique dont la sagacité s'exprime "quand la catastrophe d'Asie Mineure justifie son point de vue ». La filiation avec les chefs de guerre historiques ${ }^{93}$ s'impose naturellement quand Métaxas répond "laconiquement " à l'ultimatum italien du 28 octobre, mais l'image se nourrit aussi de la dimension démiurgique d'un Solon ou d'un Lycurgue. "Soldat, patriote, législateur, réformateur » et même " artiste» (!) ; «Figure parfaite " réunissant en lui "toutes les qualités de la "race», la postérité de Métaxas lui offrira "une éternité plus grande que celle de Périclès au siècle d'or.». On retrouve là des parentés avec la construction du mythe du chef propre aux expériences totalitaires, mais les origines " patriciennes » et le parcours politique de Ioannis Métaxas l'éloignent néanmoins d'un idéal-type mussolinien ou hitlérien ${ }^{94}$.

Les dimensions sotériologique et messianique du Guide et de son action sont également capitales. Les procédés typographiques qui attribuent au vocabulaire relatif à Métaxas des majuscules ${ }^{95}$, alimentent cette forme de « déification » du chef, renforcée par les contenus du discours de propagande qui jouent des références religieuses. Les discours opèrent un déplacement du sacré ! On place sur Métaxas bébé « quelques rameaux comme 
on l'a fait pour le Messie ", ou, il "n'est pas simplement un Être humain, pas seulement un Homme» et a surgi "dans une recréation superbe et cosmogonique [...] (comme) l'Un et Unique»! Il incarne une «Figure sacrée » qui a semé dans le peuple grec «la graine divine de Sa parole »! Alors que le christianisme est l'un des socles fondamentaux de l'idéologie du régime, la propagande avance sur une étroite ligne de crêtes entre tentation d'inspiration totalitaire ${ }^{96}$ et nécessaire respect du dogme chrétien. S'il faut un fort pouvoir d'imagination pour voir derrière le ventripotent septuagénaire et sa canne un nouveau Léonidas, la figure protectrice et rassurante du "Chef-Père » est renforcée par les inquiétudes liées à la guerre. L'orientation paternaliste est omniprésente et les innombrables références au "Père adoré ", "Père du paysan", "Véritable père " et l'inflation d'images montrant le dirigeant et des enfants en témoignent. La propagande s'emploie donc à renforcer un culte du chef qui, associé au rejet du parlementarisme et du système représentatif, tente d'établir une relation directe sans médiations entre celui-ci et le peuple. Voilà une dimension sinon fasciste, du moins populiste. Toutefois, son accomplissement se heurte à un certain nombre d'écueils : origines et parcours de Métaxas, modestie de son charisme naturel, concurrence des dévotions religieuses traditionnelles et caractère dyarchique du pouvoir imposant un partage de l'adhésion populaire avec le monarque Georges II $^{97}$. La disparition brutale du général le 29 janvier 1941 amplifie une "panthéonisation » dans laquelle la propagande n'a eu de cesse de montrer Ioannis Métaxas comme le premier soldat de la Grèce, mais aussi comme l'artisan de la création d'une Grèce nouvelle.

\section{Forger la « Troisième civilisation hellénique »} fait particulièrement acerbe : «[...] ils nous ont dénigrés et ont dit que nous étions bâtards et descendants minables d'ancêtres glorieux [...] avec du sang slave, comme Fallmerayer nous refusant la continuité avec nos aïeux [...] Ils nous ont coupés du Byzance médiéval [...] nous appelaient Grecs [...] (nous) un peuple dont le sang est racialement pur» (N.18.01.1941, p. 481). Le programme de "renaissance» - «régénération» doit prendre appui sur le meilleur de la « culture antique, grande dans le domaine de l'art, grande dans le domaine de la science", mais écarter ce qui rendait la civilisation grecque antique "boiteuse dans le domaine de la religion et plus encore en matière de développement politique $"{ }^{99}$. L'exaltation de Sparte renforce le projet de construction d'une société pyramidale dirigée par une «aristocratie politique nationale $»^{100}$. Ce rejet du principe d'égalité renvoie sinon spécifiquement aux fascismes, du moins aux idéologies contre-révolutionnaires des droites radicales en Europe.

La piété religieuse revivifiée par la guerre et ses drames interrompt la supposée dégénérescence morale et a vocation à devenir l'un des piliers identitaires du Grec 
"régénéré ». Le christianisme comme création de l'esprit grec ${ }^{101}$, n'est pas une rupture, mais un prolongement de l'esprit et de la philosophie antiques. Ainsi la prière d'une mère pour les combattants exprime-t-elle « la foi de la race grecque dans la Vierge mère de Dieu -l'Athéna Pallas, vêtue du péplos de la nouvelle religion. Ses églises sont le Parthénon " (N. 14.12.1940, p. 303). Cette construction que M. Hertzfeld qualifie d'«aménagements syncrétiques $\left.^{102} »\right)$ s'exprime parfaitement dans un récit mettant en scène Basile le Bulgaroctone venant prier après ses victoires dans un Parthénon où " métopes (et) leurs dieux antiques" cohabitent avec "l'iconostase et des figures bien connues de saints» (N. 16.11.1940, p. 138). On doit observer ici que le christianisme érigé en pilier fondamental de la "Troisième civilisation hellénique » fait obstacle à la réalisation de la «religion politique » de type fasciste.

L'accent mis sur la culture chrétienne orthodoxe trouve son expression dans l'exaltation de l'hellénisme médiéval et de l'Empire byzantin. Mais l'ambition métaxiste de construire le « Nouvel État » ou «État national » est légitimée par les nécessités de la guerre qui impliquent un renforcement de son autorité et un renoncement total aux illusions d'une "fausse liberté » au profit de la «liberté disciplinée », car «L'État a besoin de discipliner les forces nationales et de soumettre toutes les manifestations de la vie du peuple sous le joug d'un ordre nouveau et d'un but central» (N. 23.11.1940, p. 220). L'influence des théories fascistes sur la conception métaxiste de l'État apparaît ici nettement ${ }^{103}$. La guerre a pu constituer un terreau favorable à la diffusion de la "synthèse » hellénochrétienne. La propagande propose une résolution des contradictions et des confrontations entre visions eurocentristes et indigénistes, entre identités de l'Hellène et du Romios et la création du Grec nouveau, l'« Hellène chrétien ». Combinant obsession communautaire, construction d'un État national, culte du militarisme, rejet des émancipations démocratiques et combat anticommuniste, le régime peut dans son idéologie être apparenté au modèle fasciste qu'assume parfaitement Métaxas ${ }^{104}$. Néanmoins, reconnaissant le rôle privilégié de l'Église, ne pouvant s'appuyer sur un parti de masse, avec un "Führerprinzip » aux accents paternalistes et des moyens limités de diffusion de son idéologie, le régime métaxiste peut-il être assimilé à d'autres modèles autoritaires ${ }^{105}$ ?

\section{Repousser les frontières : un nouveau souffle irrédentiste}

Si la dimension impérialiste propre aux fascismes manque au régime, la guerre lui permettra de réactiver un irrédentisme inhibé depuis deux décennies. Quand en 1936, Georges Séféris écrit que «L'État grec correspond pratiquement aujourd'hui à la nation $»^{106}$, alors que la censure métaxiste interdit la publication dans la presse de toute information relative à Chypre et au Dodécanès $\mathrm{e}^{107}$, on voit que le régime poursuit, voire amplifie, les orientations géopolitiques de la Grèce depuis 1922 : la défense du territoire helladique l'emporte sur le rêve d'unification de l'hellénisme dans une grande Grèce. Correspondant à la fois aux convictions de Métaxas et aux réalités géopolitiques, le glissement d'une Grande Idée à dimension territoriale vers le projet révolutionnaire « intérieur » traduit cette orientation. Mais, avec le conflit, l'irrédentisme grec trouve un nouveau souffle.

Dans la revue datée du 9 novembre 1940 est publiée une photographie montrant "Jeunes gens et jeunes filles de l'Épire du Nord, du Dodécanèse et de Chypre dans une manifestation patriotique ». Ces manifestants enthousiastes brandissent une carte de la 
Grèce. Cette photographie et la légende qui l'accompagne mettent clairement en scène dès le début du conflit de nouveaux horizons géopolitiques pour la Grèce en guerre.

71 La Grèce des années 1920 et 1930 avait dû se résoudre à l'existence d'une Albanie englobant l'Épire du nord et à un Dodécanèse italien depuis 1912. Cependant, la guerre contre l'Italie, qui a annexé l'Albanie en 1939 et occupe toujours ces îles égéennes, réactive les prétentions grecques. Totalement refoulées par la revue depuis ses origines, les questions épirote et dodécanésienne vont à partir du déclenchement de la guerre proliférer dans ses colonnes, le passé légitimant de nouvelles ambitions. Ainsi, l'Épire est-elle présentée comme le berceau premier de l'hellénisme, peuplée « des plus anciens de tous les Grecs» (N. 18.01.1941, p. 410) et la filiation est attestée par «les historiens et les linguistes qui affirment que les Selloi qui ont aussi comme nom Elloi et les Hellènes appartiennent à la même "race» » ${ }^{108}$. C'est aussi la terre de naissance de «la » civilisation avec «Dodone, le plus ancien sanctuaire grec ». Territoire de la résistance la plus coriace de l'hellénisme chrétien à la turcocratie avec Cosmas d'Étolie, ${ }^{109}$ mais ayant aussi donné au combat pour la libération les plus grands évergètes ${ }^{110}$ et des combattants farouches et déterminés (Souliotes notamment), l'Épire démontre sa "pure grécité ». À propos du Dodécanèse, la propagande joue du ressort de l'iniquité et de la barbarie historique de la domination italienne et s'emploie à démontrer la permanence du caractère hellénique de ces îles et de l'esprit grec de résistance à l'oppression ${ }^{111}$. Le contraste est donc grand sur ces points entre le silence avant la guerre et l'inflation de références au passé pour justifier un idéal mégaloidéatique renouvelé en partie ${ }^{112}$.

Concernant Chypre, le message est plus ambigu. Il faut en effet jongler avec les rêves d'expansion, et les réalités de l'alliance britannique. C'est pourquoi à défaut de l'union ( $\varepsilon v \omega \sigma \eta)$ telle qu'on l'envisage pour Épire du Nord et Dodécanèse, la jeunesse doit se

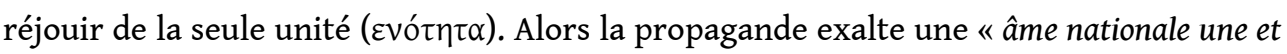
indivisible", une histoire commune puisque "Depuis la guerre de Troie, Chypre, toujours profondément grecque dans l'âme et les sentiments, a pris part à tous les combats de la Nation [...] aux cotés de la Mère-Patrie " ${ }^{113}$, mais du joug britannique et des résistances chypriotes elle ne dit rien. Un État grec incluant l'île d'Aphrodite n'est pas au programme...

73 Certaines des frontières acceptées sous la pression des grandes puissances au début du $\mathrm{xx}^{\mathrm{e}}$ siècle sont donc remises en cause. Mais, si les aspirations de la jeunesse à l'unité politique de l'hellénisme peuvent être flattées et constituer un programme mobilisateur, la Realpolitik impose des ambitions réduites, car l'alliance britannique et la bienveillante neutralité turque ne peuvent être mises en péril.

\section{Conclusion}

74 La propagande du régime propose une narration cohérente du passé à la jeunesse plongée dans une guerre. La mobilisation des éonistes repose sur une construction identitaire et communautaire: généalogie héroïque, sens du sacrifice, spiritualité supérieure. Le passé alimente la définition d'un premier cercle du Nous et disqualifie un Autre dont la différence est puissamment essentialisée.

Pendant de ces armements psychologique et idéologique, la mise en action de la jeunesse enrégimentée dans l'EON, prend des formes diverses selon l'âge et le sexe de ses jeunes membres. Le passé doit pousser chacun à remplir un devoir sacré et 
honorant le rôle qui lui est dévolu, perpétuant, mais renégociant partiellement certains stéréotypes socioculturels. En tout état de cause, la situation de guerre amplifie juvénisme viriliste et militarisation de l'existence, ainsi que caporalisation de la jeunesse qui constituent des fondements majeurs des fascismes.

Mais au-delà de la mobilisation patriotique, ayant entamé son programme de «renaissance »- « régénération » dès le 4 août 1936, le régime métaxiste trouve dans la guerre des éléments propres à amplifier et accélérer la réalisation de son ambition, la création de la Troisième civilisation hellénique. Par l'écriture d'une histoire grecque " linéaire » sous-tendue par " un principe permanent secrètement à l'œuvre sous les diverses modalités historiques de la vie grecque au cours des siècles $" 114$, expurgée de ses diverses conflictualités internes, la propagande traduit non seulement une Weltanschauung

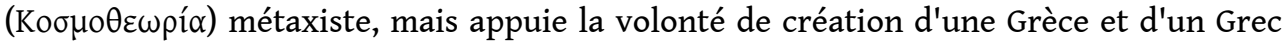
nouveau. Patrie, État, Religion, Discipline (mais aussi Monarchie, Famille) constituent les piliers de la société future dans laquelle devrait s'épanouir le Grec sous sa nouvelle

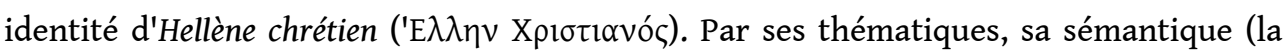
Troisième civilisation hellénique n'est pas sans rappeler le Troisième Reich), sa scénographie, son esthétique et ses emprunts à l'histoire, ainsi que sa prétention à changer l'Homme, le régime du 4 août présente de fortes analogies avec le fascisme et le totalitarisme. Néanmoins, sans doute moins marquée par la "brutalisation » de la Première Guerre mondiale, par les relégations sociales liées à la crise des années trente et le nihilisme qu'ont connus certaines sociétés européennes; la société grecque et sa " prémodernité » ne présentent-elles pas un terreau propice à certaines radicalités fasciste et totalitaire. Par ailleurs, l'absence d'un parti unique de masse, la place du christianisme et le rôle de la monarchie tempèrent la qualification fasciste du régime. De plus, le régime ne s'est pas appuyé sur certains mouvements authentiquement fascistes qui existaient à l'époque ${ }^{115}$. Enfin, si «le fascisme vient de la guerre et il y retourne $»^{116}$, c'est bien malgré elle que la Grèce est plongée dans le conflit. Ainsi le régime du 4 août occupe-t-il une place originale dans la nébuleuse des régimes autoritaires, mais de par son engagement dans la guerre et bien que partageant avec la Révolution nationale du pétainisme de nombreuses analogies, il n'en a pas la dimension de soumission à l'Axe et la coloration collaboratrice. Proche du salazarisme et sans doute du franquisme, il n'a cependant pu comme Portugal et Espagne échapper au conflit.

Nonobstant ces éléments d'un débat qui reste ouvert sur la nature du régime de Métaxas: les paradoxes d'un État fascisant combattant le fascisme, d'un régime liberticide luttant pour la liberté, s'expriment encore dans la dualité de regards portés sur celui qui est à la fois le dictateur et l'homme du «'OXl». Si sa propre expérience au pouvoir a été brève, et si son rêve d'une nation "domestiquée " et monolithique s'effondre dans la guerre civile qui déchire le pays entre 1946 et 1949, sa postérité idéologique s'exprimera néanmoins dans les discours de la junte des colonels et leur

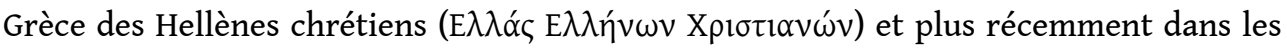

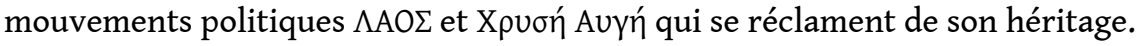




\section{BIBLIOGRAPHIE}

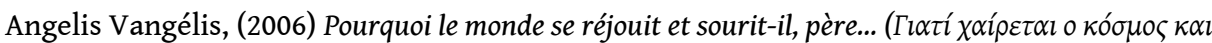

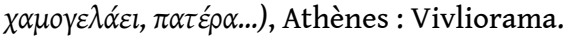

Angelopoulos Constantin, (2008), État, éducation reconstructeurs d'un passé national grec, Montpellier : PUM.

Barrachina Marie-Aline, (2011), Légitimer la participation des femmes à l'effort de guerre en zone nationale pendant la guerre d'Espagne: vers la notion de "guerre totale ", Amnis Online, 10/2011, URL : http://amnis.revues.org.

Chapoutot Johann, (2008), Le national-socialisme et l'Antiquité, Paris : PUF.

Dalègre Joëlle, (2006), La Grèce depuis 1940, Paris : L'Harmattan.

Dalègre Joëlle, (2009), « L'idéologie dans les chants de l'EPON », Cahiers balkaniques, vol. 38-39, Publications Langues O', 2008-2009, http://ceb.revues.org/749.

Delannoi G. et Taguieff P. A, (dir.), (2001), Nationalismes en perspective, Paris : Berg International Editeurs.

Dimadis Constantin A., (2003), Construction of Greek past, Gröningen: edited by Hero Hokwerda. Fleischer Hagen, (2003), La Grèce 1936-1949, (H E $\lambda \lambda \alpha ́ \delta \alpha ~ 36-49)$ Athènes : Kastaniotis.

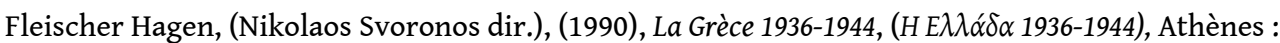
Institut culturel ATE.

Hartog François et Revel Jacques (dir.), (2001), Les usages politiques du passé, Paris : éd. EHESS.

Hertzfeld Michael, (1987), Anthropoly through the looking-glass, Cambridge University Press.

Hertzfeld Michael, (1986), Ours once more, Folklore, Ideology, and the making of Modern Greece, New York: Pella.

Kagalidou Zapheiroula, (1999), Éducation et politique, le cas du régime du 4 août, (Ek $\pi \alpha i ́ \delta \varepsilon v \sigma \eta \kappa \alpha l$

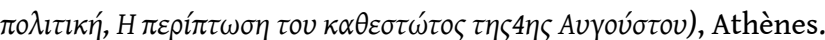

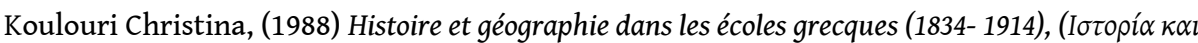

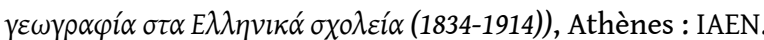

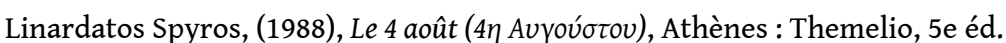

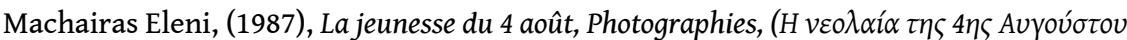

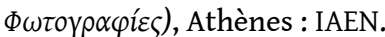

Mann, Carol, (2010), Femmes dans la guerre (1914-1945) Survivre au féminin devant et dans deux conflits mondiaux, Paris : Pygmalion.

Matard-Bonucci M. A. et P. Milza (dir), (2004), L'Homme nouveau dans l'Europe fasciste (1922-1945), Nouvelles études contemporaines, Paris : Fayard.

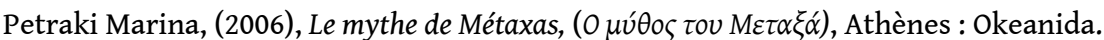

Prévélakis Constantinos, (2003), «Entre alliance et crise de confiance : la politique balkanique de la France et son échec (1938-40) », in Balkanologie, vol.VII, n 1, http://balkanologie.revues.org/ index477.htm 
Prévélakis Georges, Géopolitique de la Grèce, Éditions Complexe, 2005.

Ter Minassian Taline, (2003) Le Komintern et les Balkans, Matériau pour l'histoire de notre temps, $\mathrm{n}^{\circ}$ 71, juillet-septembre 2003.

Terrades Marc, (2005), Le drame de l'hellénisme, Ion Dragoumis et la question nationale en Grèce au début du XX siècle, Paris : l'Harmattan.

Traverso Enzo, (2001), Le totalitarisme, Le Xx siècle en débat, Paris : Éd. du Seuil.

Tsiovas Dimitris, (1989), Les métamorphoses de la nation et l'idéologie de la grécité dans l'entre-deux-

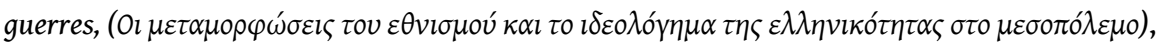
Athènes : Odyssée.

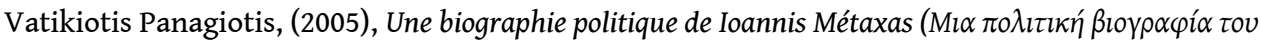

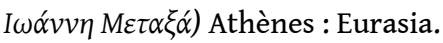

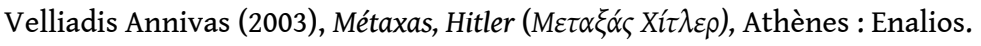

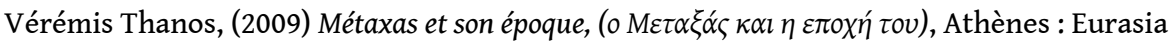

\section{NOTES}

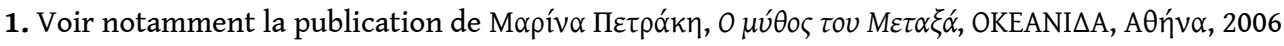

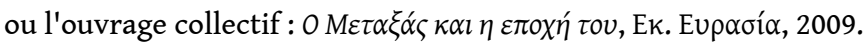

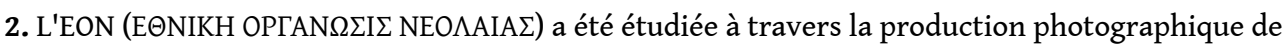

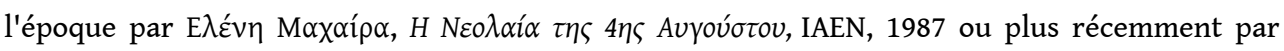

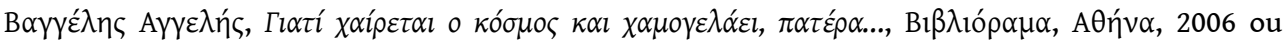
encore par Odette Varon-Vassard qui a conduit une réflexion comparative sur EON et EPON : A

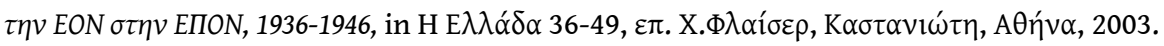

3. Ioannis Métaxas, général à la tête d'un petit groupe d'élus monarchistes et antivénizélistes au Parlement devient Premier ministre le 13 avril 1936.

4. «Lorsque la guerre éclate en 1940, il y a 2000 prisonniers politiques », Joëlle Dalègre, La Grèce depuis 1940, L'Harmattan, Paris, 2006, p. 17.

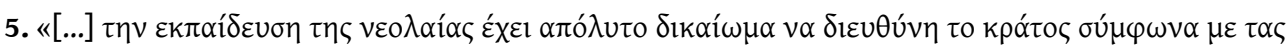

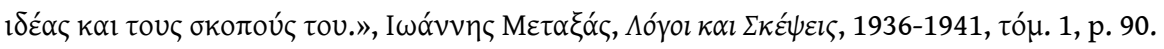

6. L'ordonnance A.N. 40 /1936 (ibid) impose la langue démotique dans l'enseignement primaire et l'année suivante est créé l'Organisme d'Édition des Livres Scolaires (OE $B$ B), A.N. 952/1937 (ibid).

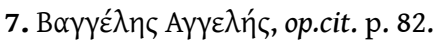

8. Ordonnance A.N. 334 /1936, Journal du Gouvernement mis en ligne par ЕӨNIКО ТҮПОГРАФЕІО, http://www.et.gr.

9. Selon les sources gouvernementales, le nombre des membres de l'EON passe de 15000 en 1937

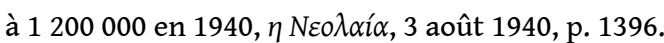

10. Le magazine survivra en effet à la disparition de Métaxas en janvier 1941 mais l'invasion allemande d'avril aura raison du régime du 4 août, de l'EON et par définition de ses publications. 11. Cette mention figure sur chacun des numéros publiés.

12. «Entre alliance et crise de confiance : la politique balkanique de la France et son échec (1938-40) », Constantinos Prévélakis, in Balkanologie, vol.VII, $\mathrm{n}^{\circ} 1$, juin 2003, http:// balkanologie.revues.org/index477.html

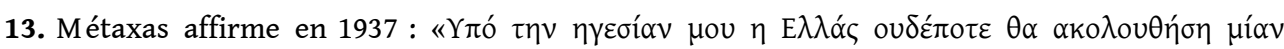

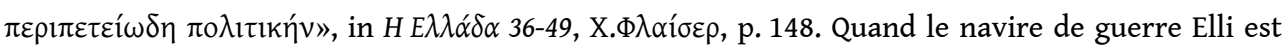
envoyé par le fond dans le port de Tinos le 15 août, alors que la torpille de fabrication italienne 


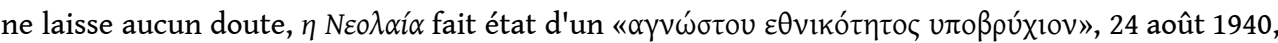
p. 1477.

14. Joëlle Dalègre, op.cit., p. 21

15. In Hartog et Revel, Les usages politiques du passé, Paris, éd. EHESS. 2001, p. 41.

16. Alexandre est avec Léonidas la figure qui revient le plus fréquemment et le premier fait l'objet d'un roman historique publié dans $\eta$ Neolaía sous la plume de Nikos Kazantzakis. On remarquera que ce feuilleton au parfum plus guerrier est toujours publié après le déclenchement des hostilités alors que l'Antigone de Sophocle se voit "expédiée " de manière résumée pour laisser davantage de place à l'actualité «engendrée par les nouvelles conditions liées à la guerre ", $\eta$ Neo $\alpha \alpha i ́ \alpha, 30$ novembre 1940, p. 245.

17. In Les usages politiques du passé, op.cit., p. 60.

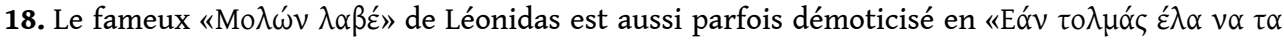

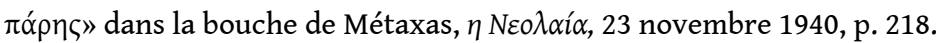

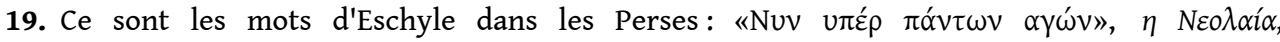

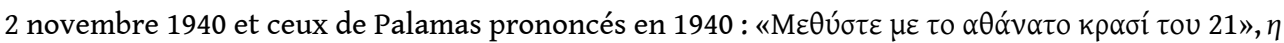

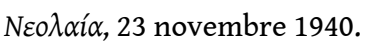

20. L'influence de Dragoumis, largement inspiré par les théories de Maurice Barrès semble s'imposer ici.

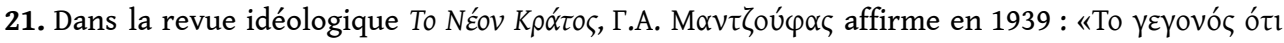

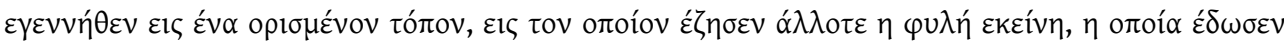

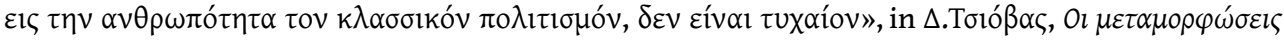

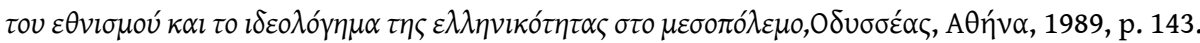

22. $\eta$ NEo $\alpha \alpha i ́ \alpha, 15$ mars 1941, p. 623. Bien sûr les impérialismes des cités-État antiques, la conquête alexandrine... sont relégués aux oubliettes de l'histoire !

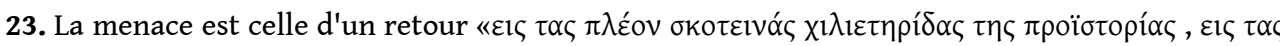

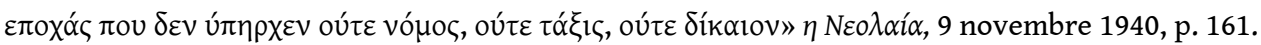

24. De nombreux récits mettent l'accent sur l'humanité des Grecs contrastant avec le comportement inhumain de l'ennemi. Cigarettes et nourriture données aux captifs italiens dessinent une grandeur d'âme grecque. Voir par exemple, l'article destiné aux Pionniers dans lequel quelques koulourakia offerts aux prisonniers deviennent un symbole civilisationnel, $\eta$

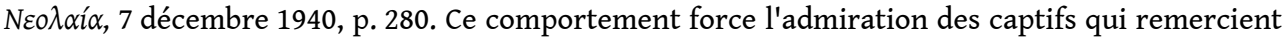

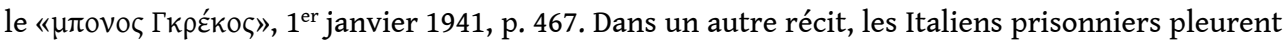
quand pensant recevoir de l'aide la Croix Rouge helvétique, ils se rendent compte qu'il s'agit de Grecs. 25 janvier 1941, p. 435.

25. En octobre 1940, la Grèce dispose d'un « armement désuet, 49 chars, 16 avions neufs [...] et ne peut aligner que 16 divisions [...] face aux 27 divisions italiennes. », Joëlle Dalègre, op.cit. . 21.

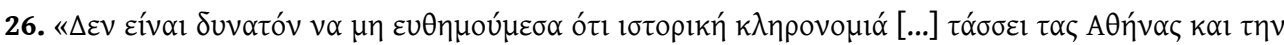

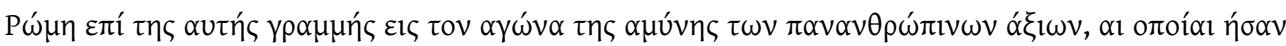

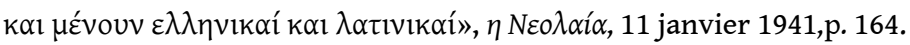

27. Ce discours présentant une continuité raciale italienne qui peuple d'innombrables articles se voit remis en cause à une seule reprise quand se pose la question des Italiens comme descendants

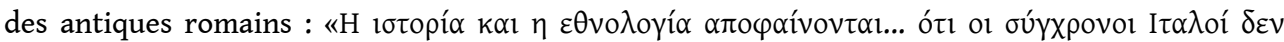

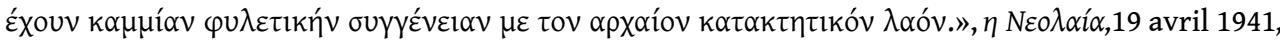
p. 724.

28. Florilège dont on peut citer quelques exemples. Les Italiens et leurs aïeux sont lâches, peureux, menteurs, rusés, indignes, sauvages, vils, inhumains, vantards, fanfarons, immoraux, injustes, hideux, répugnants, violents, haineux, gangsters ... Plus « léger » ou ironique, l'emploi permanent des termes $\mu \alpha \kappa \alpha \rho o v \alpha ́ \delta \varepsilon \varsigma$ et $\varphi \rho \alpha \tau \varepsilon ́ \lambda o l$. 


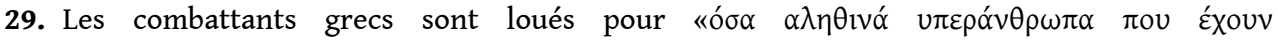

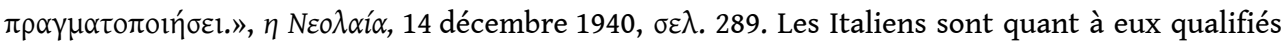

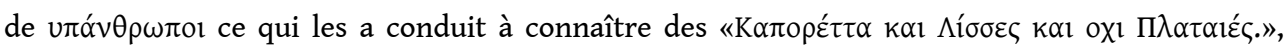
2 novembre 1940, p. 162. S'ils peuvent être victorieux ils le doivent à des méthodes infâmes n'ayant réussi à vaincre les «Éthiopiens aux pieds nus non sur le champ de bataille mais en utlisant l'ypérite. »,15 février 1941, p. 506.

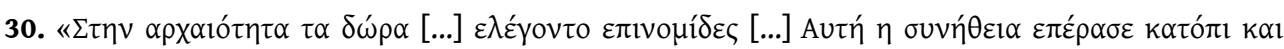

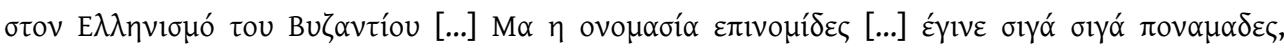

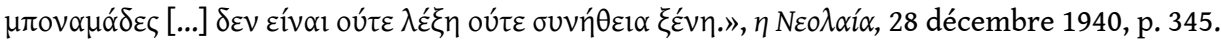

31. $\eta$ Neo $\lambda \alpha i ́ \alpha, 14$ décembre 1940, p. 290, 11 janvier 1941, p. 389, 30 novembre 1940, p. 243, 23 novembre 1940, p. 223.

32. Les Vénitiens, "plaies de l'humanité » sont maintes fois accusés de pratiques sauvages, de perfidie et lâcheté. Dans la revue où l'ironie n'a guère de place, on note dans un récit dénonçant la couardise vénitienne que ceux-ci sont invités «à voir comment se battent les Grecs

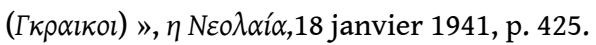

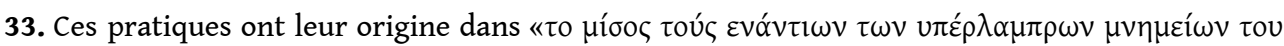

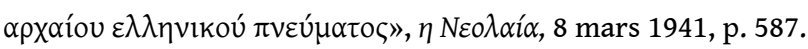

34. $\eta$ Neo $\alpha \alpha^{\alpha} \alpha, 9$ novembre 1940, p. 172. Il n'y a guère que Métaxas lui même qui tempère un peu l'italophobie en rappelant la dette grecque envers Garibaldi et Santa Rosa. 30 novembre 1940, p. 243.

35. Pour Constantin Angelopoulos : «La malédiction qui pèse sur l'autre tient à des éléments permanents comme l'appartenance phylétique, l'espace et la culture.», État, éducation reconstructeurs d'un passé national grec, PUM, Montpellier, 2008, p. 79.

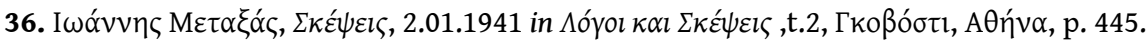

37. $\eta$ Neo $\alpha$ í $\alpha, 14$ décembre, p. 290. Le mot fascisme apparaît de manière très fréquente et

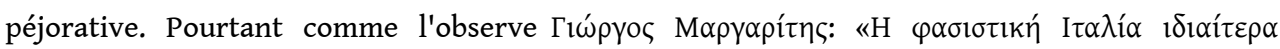

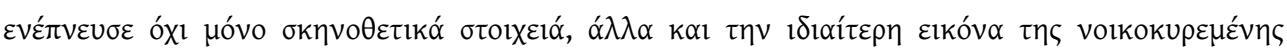

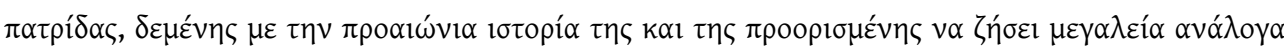

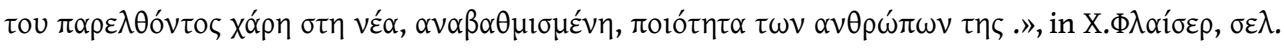

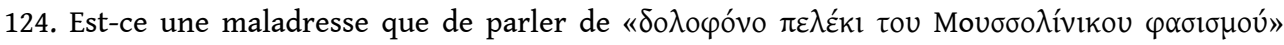
alors que la dictature métaxiste a fait d'un symbole assez comparable (labrys ou pélékis) l'emblème de l'EON ?

38. Ce sont les mots du capitaine français Capdevielle en 1926 cités par Joëlle Dalègre, La Thrace grecque, populations et territoire, L'Harmattan, p. 142, laquelle évoque également les «multiples incidents de frontière et incursions des comitadjis $"$.

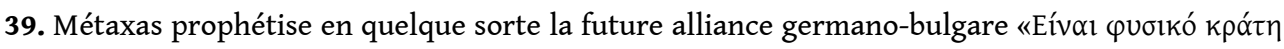

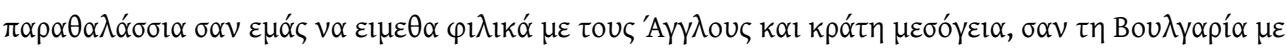

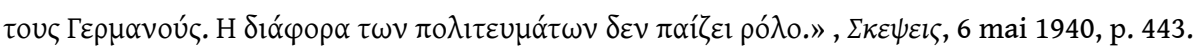

40. Marc Terrades, Le drame de l'hellénisme, Ion Dragoumis et la question nationale en Grèce au début du XXe siècle, l'Harmattan, Paris, 2005.

41. «En janvier 1934, une résolution comminatoire du secrétariat balkanique du Komintern persiste à fixer parmi ses objectifs prioritaires la création d'une république unifiée et indépendante des travailleurs macédoniens. ", Taline Ter Minassian, Le Komintern et les Balkans, Matériau pour l'histoire de notre temps, $\mathrm{n}^{\circ}$ 71, juillet-septembre 2003, p. 69.

42. Le 6 avril 1941, l'armée allemande entreprend l'Opération Marita contre la Grèce.

43. Cet officier avait tenté de négocier une reddition avec les Allemands avant le début des hostilités, voir Joëlle Dalègre, op.cit. p.23. Ceci lui vaudra d'être installé à la tête d'un gouvernement collaborateur à Athènes le 30 avril. 
44. Sitsa Karaïskaki a vécu dans l'Allemagne nazie, thuriféraire du régime hitlérien elle a

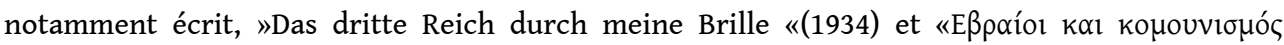
»(1934).

45. Selon Marina Pétraki, L'EON a entretenu des relations avec les organisations nazies de la

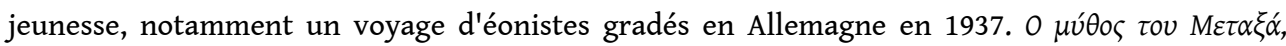
op.cit., p. 378.

46. Les relations germano-helléniques se traduisent sur le plan économique notamment par l'achat d'armes qui seront utilisées contre l'envahisseur italien et allemand! Les voyages en Grèce de Joseph Goebbels en 1936 et 1939, ou de Baldur von Shirach, le dirigeant des jeunesses hitlériennes, selon Nikos Papanastassiou, sont non officielles, touristiques en premier lieu et de

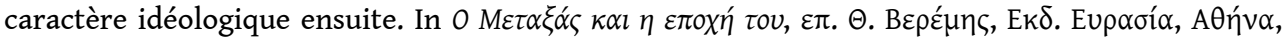
2009, p. 234. Pour d'autres comme Renate Meissner, s'appuyant sur les échanges épistolaires entre Maniadakis, le ministre de la Sécurité du régime et Himmler quant à la lutte

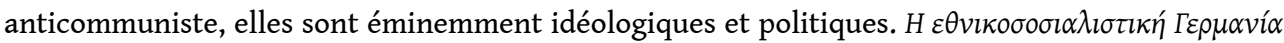

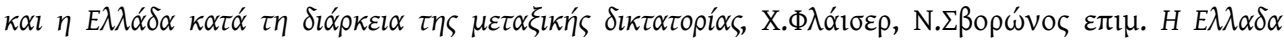

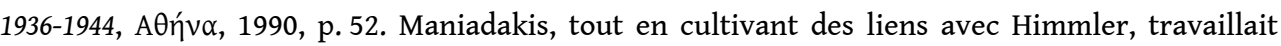

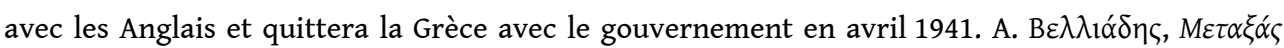

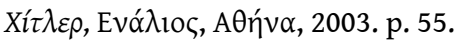

47. Le 28 avril 1941, le quotidien du NSDAP affirme : "La boucle de l'histoire universelle est bouclée, aujourd'hui dans les Thermopyles. Il y a 2500 ans, le peuple grec a résisté avec Léonidas [...] Aujourd'hui, avec nos coups puissants, nous chassons les Anglais hors d'Europe et hors de Grèce. ", in Johann Chapoutot, Le national-socialisme et l'Antiquité, PUF, Paris, 2008, p. 72, 111.

48. C'est le point de vue d'Alfred Rosenberg notamment exposé par Johan Chapoutot, op.cit., p. S73.

49. L'influence des idées de Ion Dragoumis exprimées dans Le sang ou Samothrace est perceptible. Dragoumis estime que les structures communautaires qui ont permis à l'hellénisme de perdurer n'ont pas été mises à mal par l'occupant ottoman et selon Marc Terrades: «les Turcs apparaissent non seulement comme des envahisseurs presque civilisés mais comme l'ultime rempart de l'hellénisme dans les régions directement menacées par l'activisme bulgare. », op.cit., p. 145.

50. Métaxas "has barred from schoolroom walls all patriotic pictures showing the Bloody Turk, from the public stage all the patriotic plays dramatizing the atrocities in the struggle of Greece for independence, and has ordered revision of textbooks to otherwise play down the cruelty of the Ottoman Turk.", L. Archer, Balkan Journal, An unofficial observer in Greece, Norton and cy, New York, 1944, p. 55.

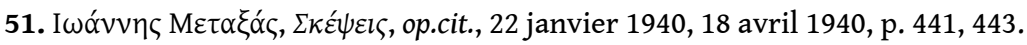

52. En 1938, Métaxas propose une alliance aux Britanniques mais il faut attendre avril 1939 pour que le Royaume-uni accepte de garantir les frontières grecques.

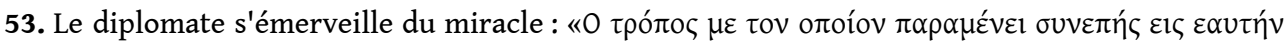

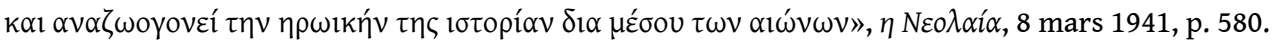

54. La notion du nécessaire sacrifice imprègne toute la phraséologie du régime et dès ses origines. En témoigne par exemple cette adresse de Métaxas aux éonistes de Colone en avril 1939,

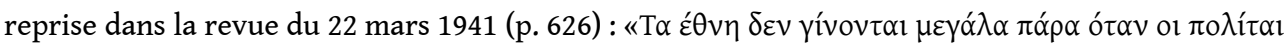

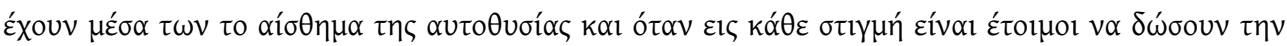
$\zeta \omega \eta ́ v \tau \omega \nu \delta i \alpha \tau \eta v \pi \alpha \tau \rho i ́ \delta \alpha . »$

55. $\eta$ Neo $\lambda \alpha i ́ \alpha, 8$ mars 1941, p. 581, 5 avril 1941, p. 678 et 12 avril 1941, p. 701 . On peut toutefois observer que l'apparition de cette rubrique est tardive puisqu'elle survient quatre mois après le début des hostilités et qu'elle n'est sans doute pas exhaustive : on peut supposer que les rédacteurs de la revue oscillent entre la valeur d'exemplarité du sacrifice des jeunes gens et les 
possibles effets traumatisants sur le jeune lecteur. Les jeunes lecteurs d' $\eta$ Neo $\lambda \alpha i ́ \alpha$ sont certes familiarisés avec la mort tant la revue présente un caractère souvent morbide mais celle-ci reste largement aseptisée et « désincarnée ».

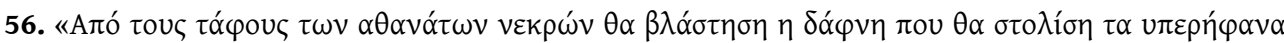

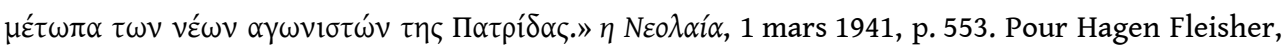
"L'épopée de 40 [...] apparaît comme une autre démonstration de la très longue histoire nationale dans laquelle l'héroïsme des défenseurs contemporains ne diffère en rien de celui des anciens. », op. cit., p. 403.

57. Parmi les très nombreux exemples, voir notamment le récit fictionnel de ce père ayant perdu

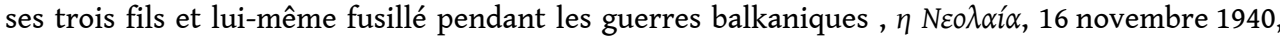
p. 197.

58. Métaxas affirme construire cette Troisième civilisation, synthèse de l'hellénisme antique et du christianisme byzantin. Il exprime souvent son désir de façonner des « Hellènes chrétiens ».

59. $\eta$ Neo $\lambda \alpha i \alpha, 25$ janvier 1941, p. 434. Les exercices physiques, la discipline, les exercices militaires et les repas communs notamment dans les structures que sont les camps d'été de l'EON traduisent bien l'imprégnation spartiate et sous couvert de liberté et autonomie sont des lieux d'apprentissage du conformisme et d'une forme d'auto-caporalisation.

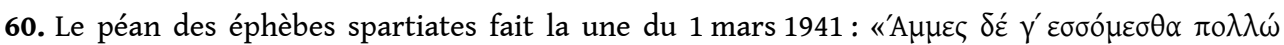

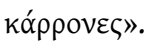

61. Parmi les récits, on retiendra celui qui voit un jeune soldat des guerres balkaniques sauvé

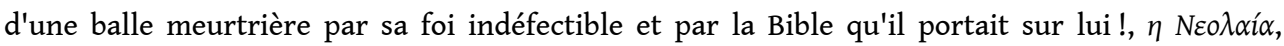
7 décembre 1940, p. 270.

62. Selon Carlton J.Hayes, cette combinaison du national et du religieux conduit au degré le plus élevé du nationalisme, Nationalism: a Religion, New York, Macmillan, 1960, p. 10, cité par Paul Zawadzki, Le nationalisme comme religion séculière, in Nationalismes en perspective, dir. G. Delannoi et P. A. Taguieff, Berg International Editeurs, Paris, 2001, p. 266.

63. Ce credo est exprimée dans la revue datée du 26 novembre 1938, p. 227.

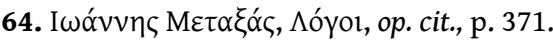

65. L'article évoque une manifestation de l'EON dans un théâtre d'Athènes à l'occasion de la fête

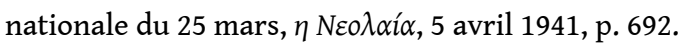

66. À l'occasion du Nouvel An, en donnant quelques uns de ses jouets à ses petits voisins dont le père est sur le front, le petit éoniste réjouira ces enfants et ainsi leur papa qui « $\theta \alpha \pi 0 \lambda \varepsilon \mu \alpha \eta_{\eta} \mu \varepsilon$

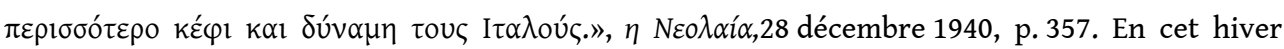
1940-1941, le Père Noël est d'ailleurs "vêtu de l'uniforme kaki » et dans sa " hotte pleine de chaussettes, de gants, de pullover...», pas de place pour les cadeaux des enfants..., p. 356. Dans une version iconographique sur la couverture du magazine, la hotte est remplie de prisonniers italiens!

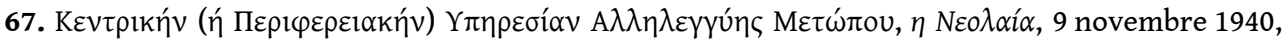
p. 162.

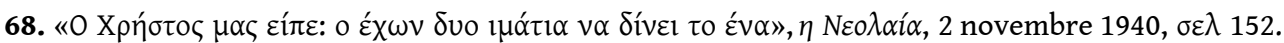
Les éonistes sont appelés à envoyer 70 drachmes afin que les soldats reçoivent un colis pour les étrennes. De plus, la revue publie de nombreux clichés montrant la collecte enthousiaste de marchandises par les Phalangistes. Voir par exemple $\eta$ Neo $\lambda \alpha i ́ \alpha, 16$ novembre 1940. Ils sont aussi

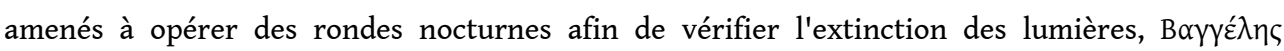

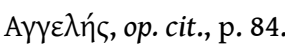

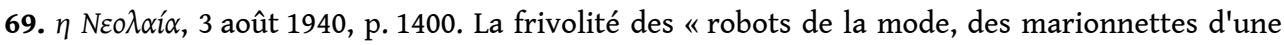

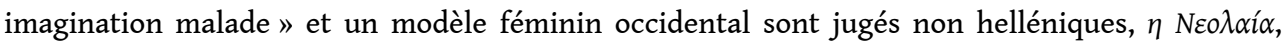
17 décembre 1938, p. 345. Par ailleurs, Métaxas dissout les organisations féministes poursuivant en cela l'entreprise réactionnaire déjà amorcée puisque des mesures législatives avaient restreint 


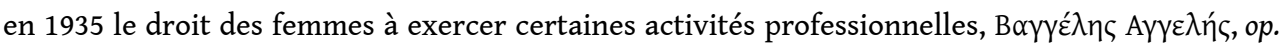
cit., p. 188.

70. $\eta$ Neo $\lambda \alpha i ́ \alpha, 26$ novembre 1940, p. 240. D'abord très brièvement baptisées «La reine de la maison », les pages destinées au lectorat féminin ont pour titre « La page de nos filles ». Elles sont composées en général d'un article idéologique de Sitsa Karaïskaki et de multiples conseils et injonctions domestiques. L'archétype féminin qui s'impose est celui de la ménagère et de la mère de famille.

71. Culte du sport et du corps sain qui recouvre une préoccupation qui, si elle est puissamment idéologisée dans les régimes totalitaires, est aussi à l'oeuvre aussi dans les sociétés occidentales.

72. Le débat existe entre historiens des fascismes. Pour certains «l'intégration dans des organisations de masse ayant pu jouer comme facteur d'émancipation en dépit des idéologies conservatrices qui les régissaient. ", Marie-Anne Matard-Bonucci, L'Homme nouveau dans l'Europe fasciste (1922-1945), M. A. Matard-Bonucci et P. Milza (dir), Nouvelles études contemporaines, Fayard, Paris, 2004, p. 9.

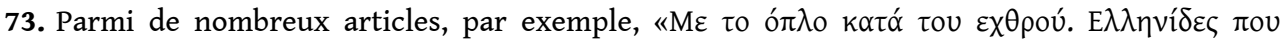

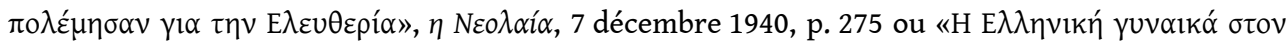

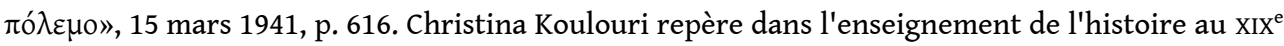
et début $\mathrm{du} \mathrm{Xx} \mathrm{x}^{\mathrm{e}}$ siècle la présentation des Souliotes comme porteuses de vertus masculines qui en

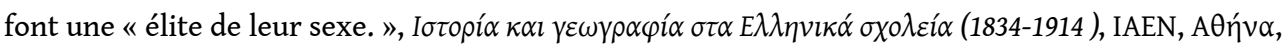
1988, p. 85.

74. Les femmes ont joué un rôle crucial dans les défaites italiennes, leur action est comparée à celle des femmes de Sparte qui en 272 av. J.C. auraient joué un rôle de soutien à leurs hommes en armes.

75. M.A. Barrachina repère pour la guerre civile espagnole le même type de " conflit entre deux idéaux : l'idéal de la phalangiste active, courageuse, responsable, capable de se mouvoir dans l'espace de la guerre [...] (auquel s'oppose) un idéal phalangiste de la femme, mue par ses qualités et fonctions féminines spécifiques. ", ainsi que la " contradiction entre le devoir domestique exigé par la tradition catholique et patriarcale et le devoir national qui, en temps de guerre, requiert la présence des femmes dans l'espace public.", Légitimer la participation des femmes à l'effort de guerre en zone nationale pendant la guerre d'Espagne: vers la notion de "guerre totale, Amnis Online, 10/2011 URL: http://amnis.revues.org. Ces hésitations s'observent dans la ligne vantée aux jeunes lectrices.

76. Le rôle des femmes dans le sacrifice consenti des Trois cents est mis en avant. «(O

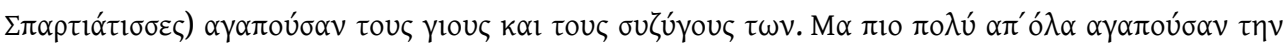

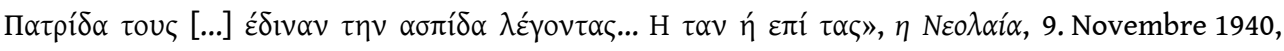

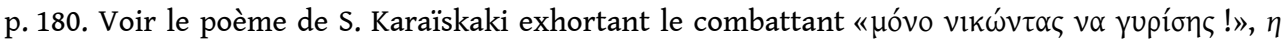

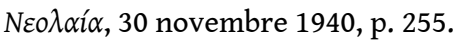

77. $\eta$ Neo $\lambda \alpha i \alpha, 11$ janvier 1941, p. 400. «Tricotez! » le mot d'ordre peuple d'innombrables pages de la revue mais le tricot est présenté comme une activité naturellement féminine que ne peut pratiquer un garçon, $\eta$ NEo $\lambda \alpha i ́ \alpha, 28$ décembre 1940, p. 357.

78. On doit à Carol Mann l'expression d'« utérus nationalisé ", le nazisme en a poussé la logique jusqu'à des extrêmes mais cette tendance apparaît avec la Première Guerre mondiale, Femmes dans la guerre (1914-1945) Survivre au féminin devant et dans deux conflits mondiaux, Pygmalion, Paris, 2010.

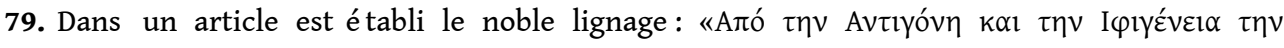

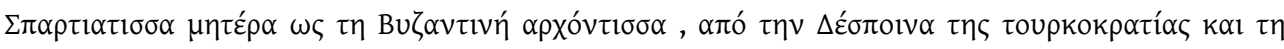

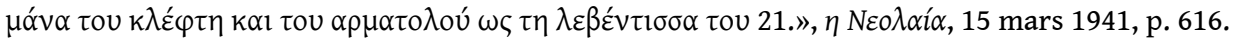


80. L'Organisation Unie Panhelladique de Jeunesse, émanation du Front National de Libération (EAM) a été créée le 23 février 1943.Voir Joëlle Dalègre, «L'idéologie dans les chants de l'EPON », Cahiers Balkaniques, vol. 38-39, Publications Langues O', 2008-2009, http://ceb.revues.org/749

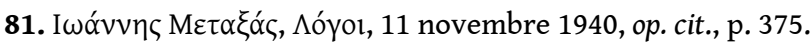

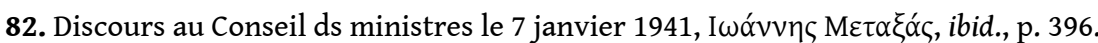

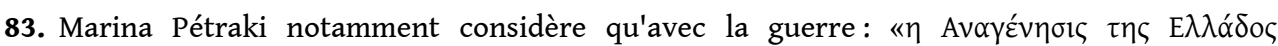

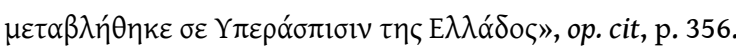

84. $\eta$ Neo $\lambda \alpha i ́ \alpha$, 1 mars 1941, p. 556. La déclaration de Séraphim Maximou, cadre dirigeant et ex député du Parti communiste, fait écho à l'appel de Zachariadis à s'unir derrière Métaxas contre l'ennemi italien.

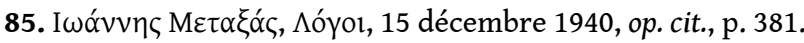

86. Des théoriciens du régime N. D. Koumarou et G. A. Mantzoufas dénoncent « la monstrueuse

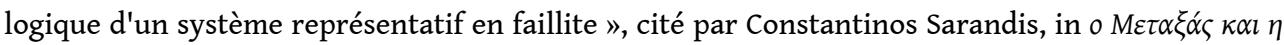

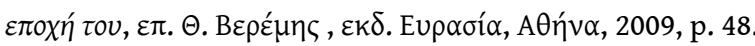

87. La seule et unique occurrence du mot $\Delta \eta \mu o \kappa \rho \alpha \tau i ́ \alpha$ dans la revue renvoie à l'attrait que

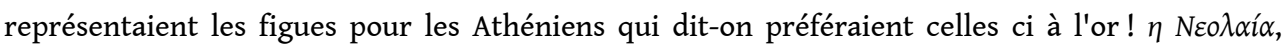
8 mars 1941, p. 587.

88. À propos de la conquête romaine : «E

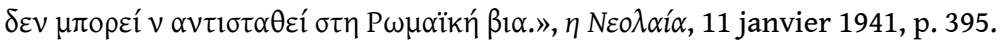

89. $\eta$ NEo $\alpha \alpha i \alpha, 9$ novembre 1940, p. 164. Noter comment le régime négocie la question des rébètes qui, réprimés à partir de 1937 avec l'interdiction des amanadès, retrouvent "droit de cité » en composant des chansons patriotiques et farouchement antimussoliniennes.

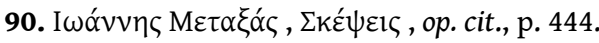

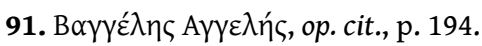

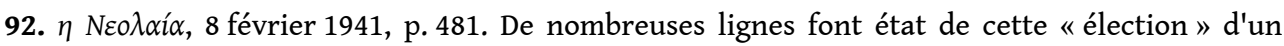

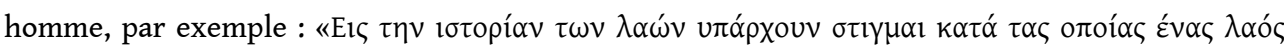

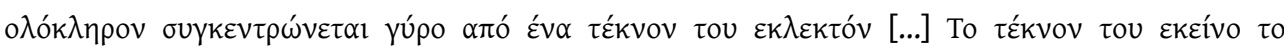

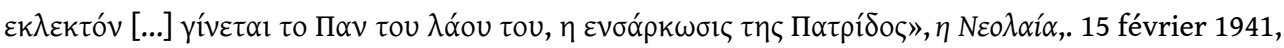
p. 509.

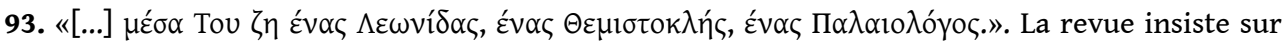
les qualités militaires de Métaxas. Néanmoins, le général refusa toujours de revêtir son uniforme...

94. À propos des totalitarismes, Enzo Traverso reprend l'idée d'Arno J. Meyer parlant de « contre-élites politiques » considérant « le caractère plébéien de leaders [...] qui comme Hitler ont découvert leur talent politique dans la rue ou, comme Mussolini, viennent de la gauche, où ils ont fait l'expérience des mouvements de masse ", Le totalitarisme, Le $\mathrm{XX}^{e}$ siècle en débat, Seuil, Paris, 2001, p. 15.

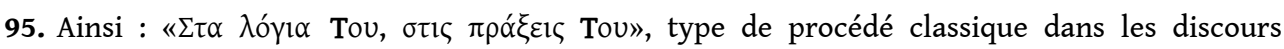
religieux.

96. Ces procédés sont bien identifiés dans les régimes totalitaires dans lesquels la « religion politique» se substitue aux religions traditionnelles, les chefs étant dotés de pouvoirs surhumains et surnaturels.

97. La répartition des rôles entre Métaxas et Georges II s'exprime dans un credo affirmé dans la

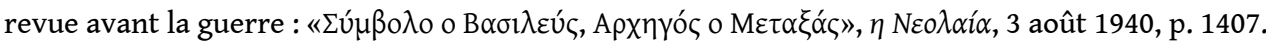

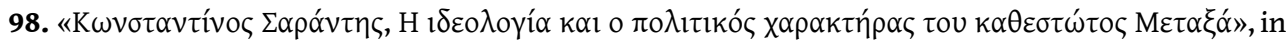

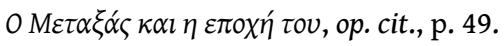

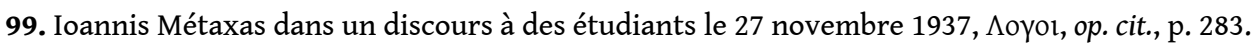

100. Ces termes dans la revue To Néov Kpátoৎ en 1937 sont cités par Zafeiroula Kagalidou,

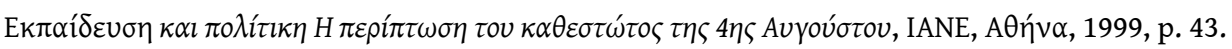




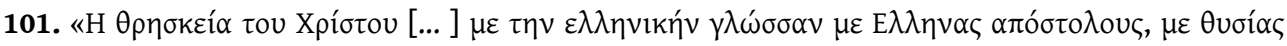

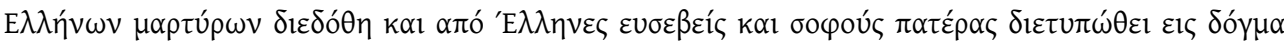

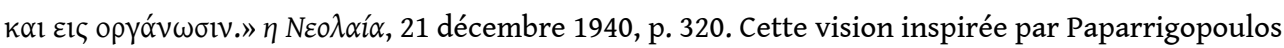
est exposée par Marc Terrades : « L'élément grec a été, au sens socratique du terme, l'accoucheur du christianisme. Inversement, en accueillant le christianisme, l'Hellénisme a accompli sa propre essence ", Le drame de l'Hellénisme, op. cit., p. 221.

102. In Les usages politiques du passé, op. cit., p. 44.

103. «Le libéralisme met l'État au service de l'individu; le fascisme réaffirme l'État comme la véritable réalité de l'individu », Giovanni Gentile, cité par Enzo Traverso, op. cit., p. 21.

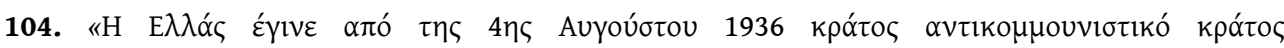

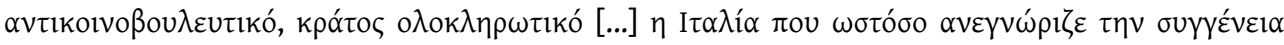

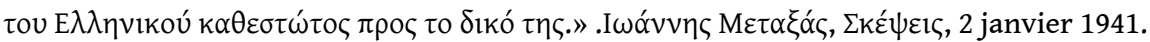

105. La classification du franquisme, du salazarisme, du pétainisme est sujette à de nombreux débats.

106. Cité par Constantin A. Dimadis, Construction of Greek past, éd Hokwerda, Gröningen, 2003, p. 266.

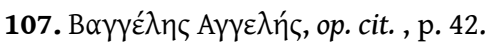

108. $\eta$ Neo $\lambda \alpha i ́ \alpha, 23$ novembre 1940, p. 227. La filiation aïeux-contemporains est renforcée : «H

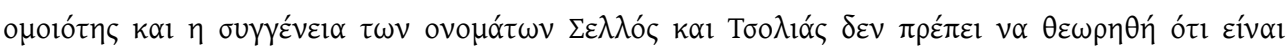

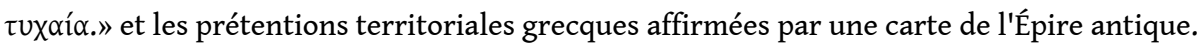

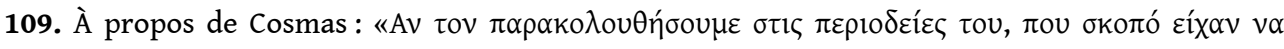

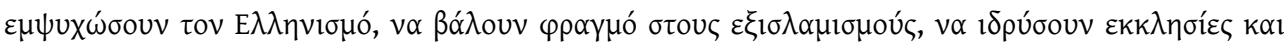

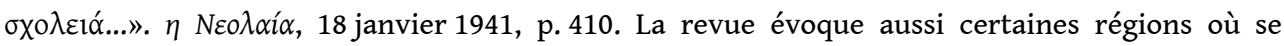

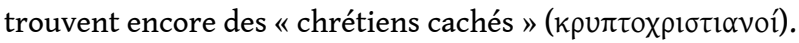

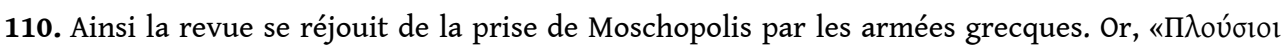

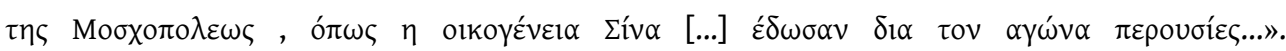
22 février 1941, p. 536.

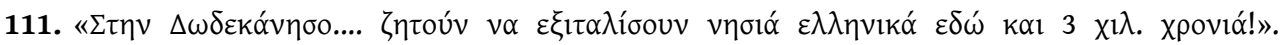
14 décembre 1940, p. 288 Le "Superbe "Non" d'Hippocrate de Kos » à Artaxerxès le Perse s'ajoute

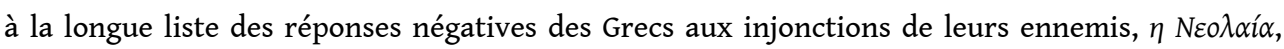
5 avril 1941, p. 679.

112. Nous et notre apparaissent très fréquemment à propos du Dodécanèse et de villes épirotes comme Himara, Argyrokastro, Korytsa etc... Par ailleurs cet axe géopolitique redéfini se manifeste de manière limpide chez Métaxas qui, dans un discours pour le troisième anniversaire

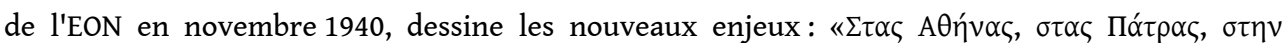

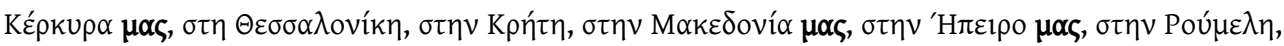

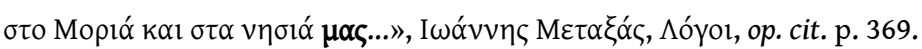

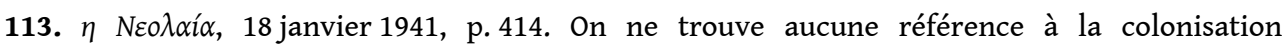
britannique et au soulèvement de 1931. En revanche le combat mené par les Chypriotes pour la défense de la « Mère-Patrie » est salué : courage des Chypriotes sur le front, tricot des femmes et générosité des dons.

114. Marc Terrades, op. cit., p.16. Il voit là la construction d'un mythe qu'on doit à Paparrigopoulos.

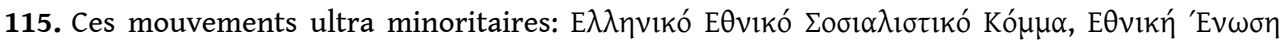

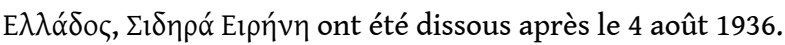

116. Pascal Ory, Du fascisme, Perrin, Paris, 2010. 


\section{RÉSUMÉS}

Il s'agit d'examiner la façon dont passé et histoire sont instrumentalisés à des fins de propagande par le régime du 4 août en Grèce, alors que le pays affronte les attaques italienne puis allemande depuis le 28 octobre 1940 et ce jusqu'à la capitulation d'avril 1941. Ce sont spécifiquement la revue La Jeunesse ( $\eta N \varepsilon$ Noג任) (publication de l'Organisation nationale de la Jeunesse-EON), et les discours en direction de celle-ci, qui sont analysés. La propagande construit sur les ressources offertes par le passé un discours visant à disqualifier l'ennemi, à renforcer l'unité nationale et à exiger la mobilisation de tous. Mais ces mêmes discours dépassent l'objectif de défense de l'indépendance nationale pour amplifier le programme de « renaissance " - « régénération » de la société entrepris par le régime avant le déclenchement du conflit. Un premier axe conduit à exposer comment le passé éclaire la lecture des enjeux du conflit et alimente la définition du Nous face à l'Autre. Le second explore la façon dont des "modèles " historiques assignent à chacun, selon son âge et son sexe, un rôle particulier dans la guerre. Le troisième montre comment le contexte spécifique de la guerre et l'« écriture » de l'histoire qui en découle, ont pu constituer une «opportunité » pour le Régime du 4 août dans la réalisation d'objectifs dont la dimension totalitaire sera interrogée.

This article aims at studying how the past and history were instrumentalized in the purpose of propaganda by the Greek regime of the 4th of August while the country had undergone Italian and German attacks since 28th of October 1940 until its capitulation in April 1941. The speeches to the youth will be analyzed through the study of the magazine The Youth ( $\eta$ Neo $\lambda \alpha i$ í $\alpha$ ) (publication of the National Organization of the Youth). Drawing its inspiration from the resources of the past, propaganda constructs its own speech, aimed at bringing discredit on the enemy, as well as strengthening national consensus and eventually calling on everyone to mobilize. But these speeches also go beyond the objective of the sole defense of independence, in order to amplify its program of "rebirth" and "regeneration" of the society formerly set up by the regime before the conflict began. A first approach leads to show how the past highlights a good understanding of the stakes of the conflict and thus enriching the definition of the "Us" vs the "Other". The second explores the way historic models assign each one to a specific role in the war, according to their age and sex. The third shows how the specific context of the war and the "writing" of history which stems from it, could give an opportunity for the regime to reach objectives whose totalitarian dimension will be questioned.

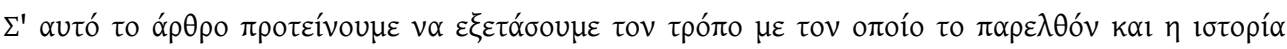

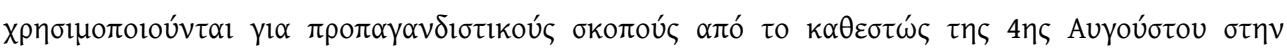

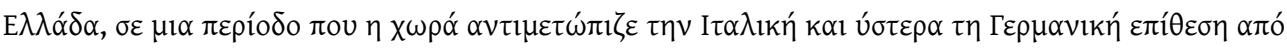

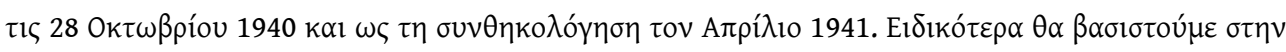

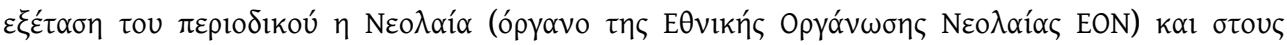

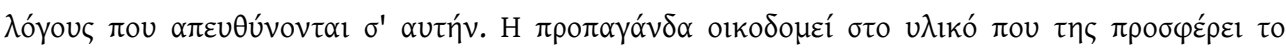

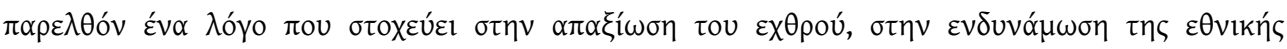

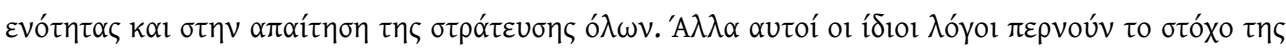

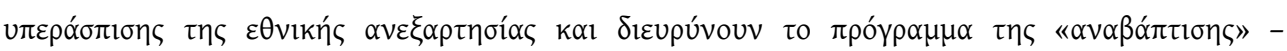

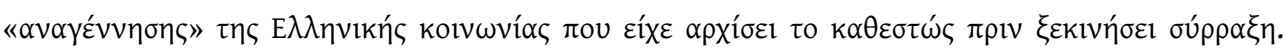

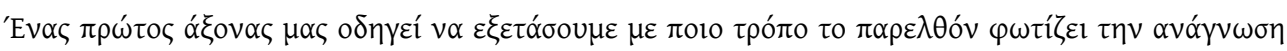

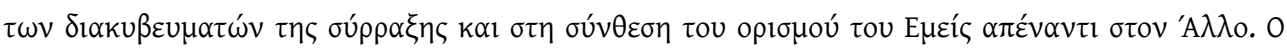

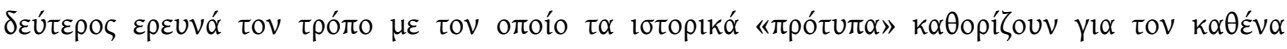

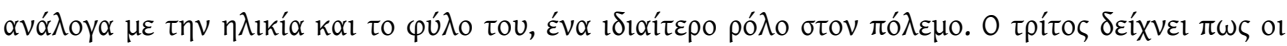




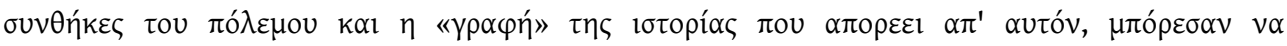

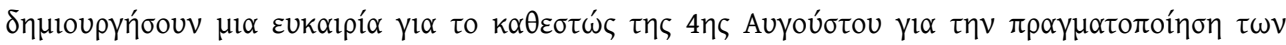

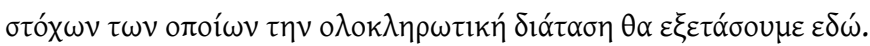

\section{INDEX}

Index géographique : Grèce

motsclestr Metaxas Ioannis (1871-1941), Gençlik örgütü, Propaganda, Gençler için basın, Tarih öğretiminde, Yunanistan, Metaxas diktatörlüğü, Tarih, Basın Tarihi

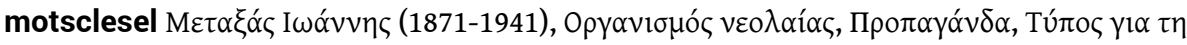

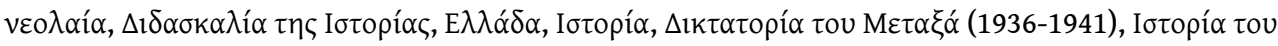

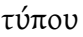

motsclesmk МЕТАКСАС ЈОАНИС (1871-1941), МЛАДИНСКА ОРГАНИЗАЦИЈА, ПРОПАГАНДА, ПРИТИСНЕТЕ ЗА МЛАДИ, НАСТАВАТА ПО ИСТОРИЈА, ГРЦИЈА, МЕТАКСАС ДИКТАТУРА, ИСТОРИЈА, ИСТОРИЈА НА ПЕЧАТОТ

Thèmes : Histoire, Histoire de la presse

Mots-clés : organisation de jeunesse, EON, propagande, EPON, endoctrinement, Metaxás Ioannis (1871-1941), presse pour la jeunesse, Metaxás Ioannis (1871-1941)

Index chronologique : dictature de Métaxas (1936-1941) 